
UNIVERSITAT DE GIRONA

Resumen: Se estudia cuál es la percepción de los estudiantes de Derecho en modalidad presencial, a partir del empleo de una herramienta wiki para la realización de una investigación en grupo. En particular, se presta atención a los factores que pueden explicar la respuesta negativa de algunos estudiantes en relación con el aprendizaje cooperativo y el sentimiento de frustración experimentado por ellos, y se formulan algunas propuestas de mejora.

Palabras clave: Frustración; Aprendizaje cooperativo; CSCL; Derecho; Wiki. 


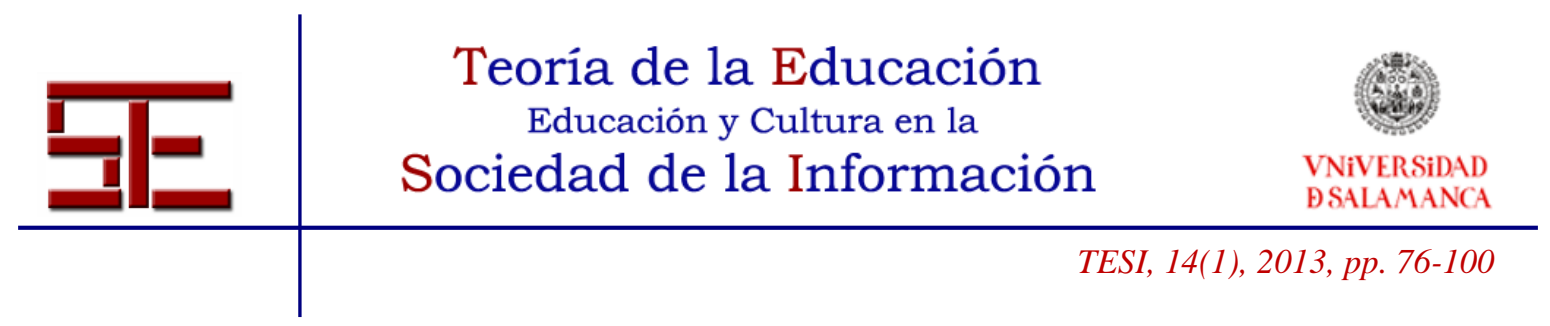

STUDENTS' PERCEPTIONS ABOUT THE USE OF WIKI. ANALYSIS OF AN EXPERIENCE OF COLLABORATIVE LEARNING AT THE UNIVERSITY OF GIRONA

Abstract: This paper focuses on frustration experienced by law students as a result of using wikis to carry out a group investigation assignment at an on-site university. The reasons which may have contributed to such a negative response by the students are explored and some proposals for improvement are presented.

Key words: Cooperative learning; Frustration; CSCL; Law; Wiki.

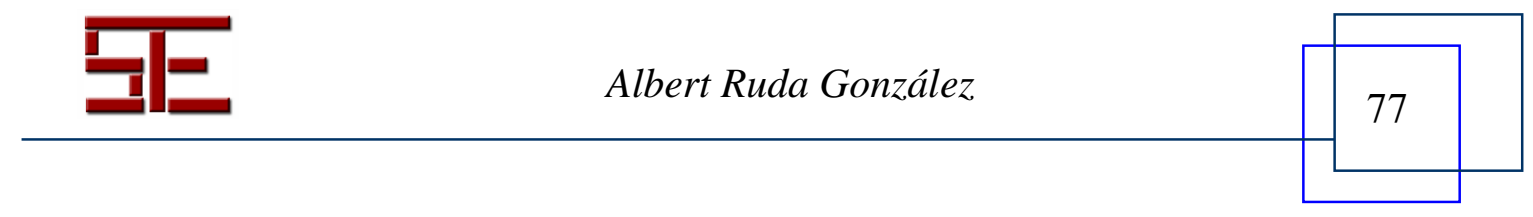




\begin{tabular}{cc} 
Teoría de la Educación \\
$\begin{array}{c}\text { Educación y Cultura en la } \\
\text { VNiVERSIDAD } \\
\text { BSALAMANCA }\end{array}$ \\
\hline TESI, 14(1), 2013, pp. 76-100
\end{tabular}

\section{PERCEPCIÓN DE LOS ESTUDIANTES SOBRE EL USO DE WIKI. ANÁLISIS DE UNA EXPERIENCIA DE APRENDIZAJE COLABORATIVO EN LA UNIVERSITAT DE GIRONA}

Fecha de recepción: 02/12/2012; fecha de aceptación: 26/01/2013; fecha de publicación: 28/02/2013

Albert Ruda González

ruda@elaw.udg.edu

Universitat de Girona,

\section{1.- INTRODUCCIÓN ${ }^{1}$}

El presente trabajo describe una experiencia de utilización de wikis para la realización de un trabajo de investigación en grupo por estudiantes de Derecho en una universidad presencial. Una encuesta realizada tras la actividad reflejó que buena parte de los estudiantes decían haberse sentido frustrados. A continuación se exponen los factores que, en la opinión de los alumnos, pueden haber causado esa frustración.

Fundamentalmente, la experiencia se refiere a la impartición de una asignatura en una carrera de Derecho. En particular, el profesorado ofreció las wikis como plataforma para realizar un trabajo de investigación en grupo, que formaría parte de la nota de prácticas de la asignatura. Dado el peso de dicha actividad en el conjunto de la nota, puede hablarse de un trabajo colaborativo elaborado en modalidad de aprendizaje mixto o blended learning. El trabajo analiza cómo este aspecto puede haber influido en la respuesta de los estudiantes y formula propuestas de mejora.

\section{2.- REFERENTES TEÓRICOS}

La literatura subraya la necesidad de conocer con más exactitud los fundamentos, los efectos y los mecanismos concretos que explican efectos positivos cuando se emplean

\footnotetext{
${ }^{1}$ Trabajo elaborado en el marco de una Ayuda MQD de la AGAUR (2010MQD00017) para el período 2011-2012, de la que es investigador principal el autor. Este agradece a las profesoras Montse Guitert Catasús (RACEV/Edul@b, UOC) y Margarida Romero (UOC) sus observaciones a versiones previas de este trabajo.
}

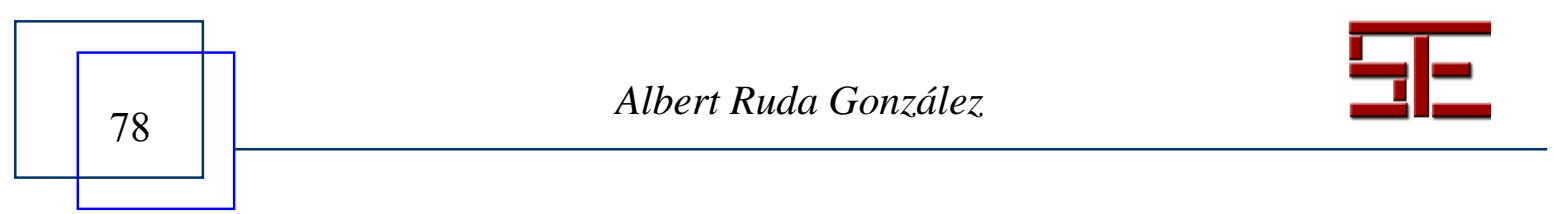




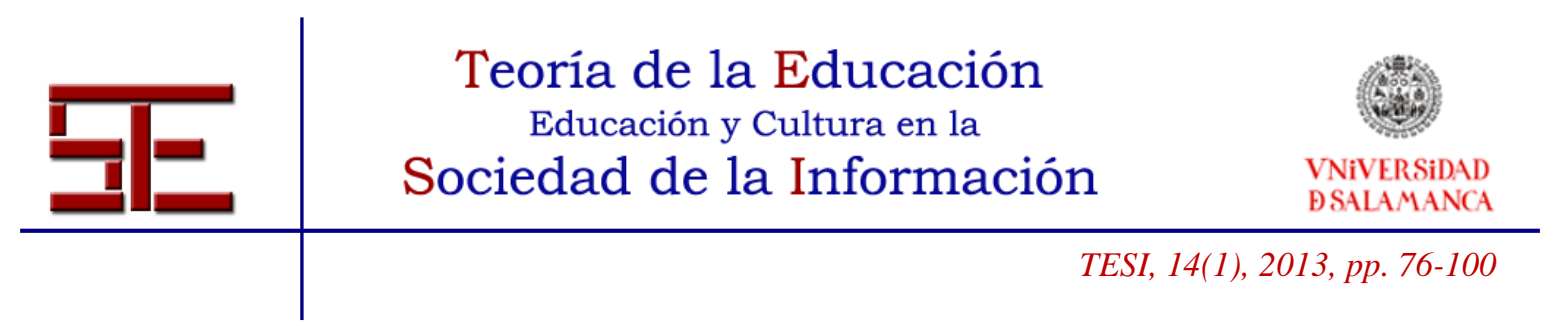

métodos de aprendizaje colaborativo (Goikoety Pascual, 2002). En especial, aunque una asignatura se haya estado impartiendo durante años en la modalidad presencial, el tránsito hacia su impartición en línea puede provocar situaciones de frustración de los estudiantes (Sela, 2010). Esta se refiere a determinadas condiciones externas a un sujeto, que le impiden conseguir un objetivo que persigue, pero también a la reacción del organismo a ese acontecimiento (Berkowitz, 1989). La frustración es un sentimiento que ocurre por no poder alcanzar un objetivo al cual se aspiraba o se deseaba. Consiste en la reacción producida al imposibilitar a un organismo acceder al objetivo de su conducta motivada (Chóliz, 2004).

Los factores de la frustración tienen en parte que ver con la psicología del individuo. Estos se pueden dividir en elementos intrapsicológicos y interpsicológicos. Ya Freud, al referirse a la frustración, señaló que puede deberse tanto a factores internos como externos. En cuanto a los internos, se trata de una respuesta del individuo que no se acompasa con un cambio de circunstancias. Cuando aparece el obstáculo que impide que el estudiante alcance su objetivo, el mismo estudiante no se adapta bien y esto genera frustración (sobre esta perspectiva, se puede ver Bessiere et al., 2004). Los factores intrapsicológicos se refieren a aspectos internos, como la propia expresión da a entender. En particular, la personalidad del estudiante, sus motivaciones, y otros aspectos de carácter subjetivo o interno. El estudiante puede estar poco preparado, sea por falta de conocimiento, falta de habilidad o falta de capacidad física. Este aspecto es de primer orden, ya que si la motivación del alumno resulta afectada, es menos probable que quiera emprender un curso nuevo, especialmente online. La literatura pone de relieve que el valor que se atribuye a la tarea (task value), la autoeficacia (self-efficacy) [relativa a la confianza del individuo en sus propias capacidades], el aburrimiento y la frustración funcionan como indicadores, en un sentido estadístico, de que el estudiante no quedará satisfecho con un curso determinado (Artino, 2008). Especialmente, la literatura se refiere a la auto-eficacia como una condición interna que determina el estado afectivo del estudiante, y que puede tener un impacto sobre la frustración cuando algún obstáculo le impide alcanzar su objetivo (Bessiere et al., 2004).

Los segundos tienen que ver con los aspectos exteriores de la psicología, en el sentido de que la persona interactúa con otros individuos de una forma determinada. Si por ejemplo los otros individuos se lo ponen difícil y el estudiante tiene poca confianza en sí mismo, es más fácil que se sienta frustrado. También, es necesario que el estudiante no se aísle (Borges, 2005). La situación también puede producirse porque el profesor no fomente la

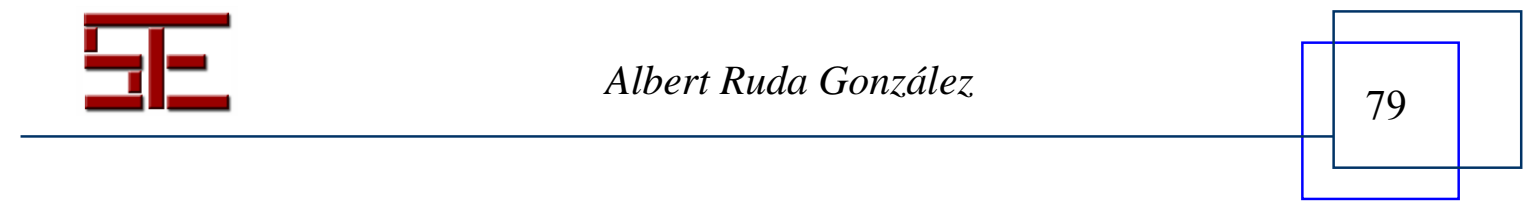




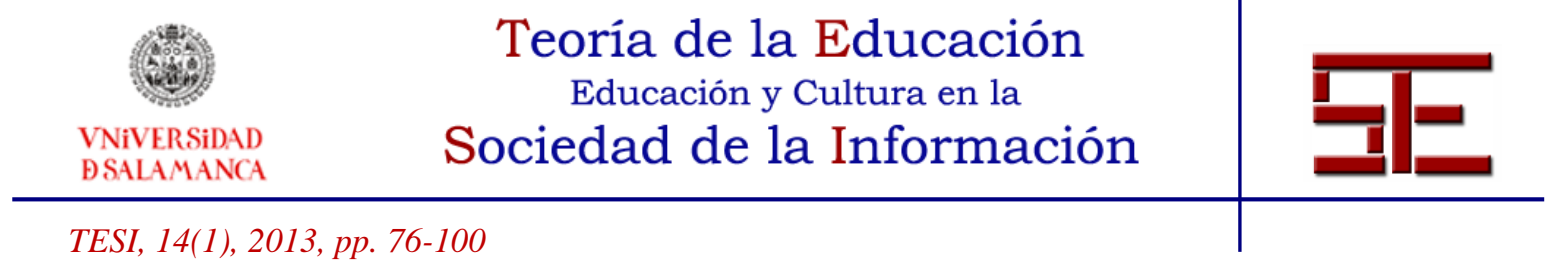

interacción o la colaboración (nuevamente, Borges, 2005). Posiblemente también habría que incluir el hecho de que haya una distribución desigual del trabajo dentro del grupo (free-riders). Ese desequilibrio lleva a una percepción de injusticia, que condiciona la actitud del estudiante (Burdett; Hastie, 2009).

El otro grupo de elementos tiene que ver con factores completamente externos al individuo, y serían aquellos que determinan la situación o contexto en que se encuentra. Estos elementos se podrían calificar como "situacionales" (Bessiere et al., 2004). Incluyen el grado de compromiso con los objetivos, medidos como la importancia que se atribuye a la tarea, la intensidad del deseo de obtener los objetivos, medidos como expectativas de anticipación, y la severidad de la interrupción, medida como tiempo perdido. Como parte del contexto tenemos el elemento tecnológico, ya que define el marco para el aprendizaje. La tecnología es fundamental en el aprendizaje online, y también juega un papel considerable en el aprendizaje presencial o semipresencial. En particular, los estudiantes pueden percibir como una dificultad añadida el hecho de tener que comunicar online (que también puede representar un reto para los profesores, como señala Kyung, 2007). Similarmente, el estudiante puede, antes de haber iniciado la actividad, preconcebir la tecnología como un impedimento, más que como una ayuda para completar su tarea (Bessiere et al., 2004). También puede suceder que haya un cambio de circunstancias que requeriría un cambio correlativo a la interfaz que el estudiante usa, pero que por alguna razón no sea posible llevarlo a cabo, lo que constituiría un factor externo fuente de insatisfacción. Otros aspectos externos que podrían tener un impacto sobre la frustración del estudiante serían elementos como las barreras sociales o jurídicas, el entorno físico o el comportamiento de otras personas (Bessiere et al., 2004). Especialmente, puede influir la institución, que quizá ofrece una ayuda técnica deficiente, no prepara adecuadamente al profesor, organiza mal el curso, no da formación preliminar al estudiante, ni le orienta o ayuda, le ofrece expectativas irreales, o contribuye a su sobrecarga de trabajo (Borges, 2005). En concreto, la literatura sugiere que los profesores no hacen lo suficiente para facilitar las experiencias de trabajo en grupo de los alumnos (por ejemplo, porque no discuten con ellos las dinámicas de grupo y similares, véase Chapman y Van Auken, 2001).

Aparte de lo anterior, hay que recordar que el curso era fundamentalmente presencial, pero que el trabajo en grupo era virtual. En los cursos online hay varios factores más propensos a generar frustración: el hecho de que la comunicación sea virtual y asíncrona hace que a veces se retrasen las respuestas de los interlocutores, lo que puede causar

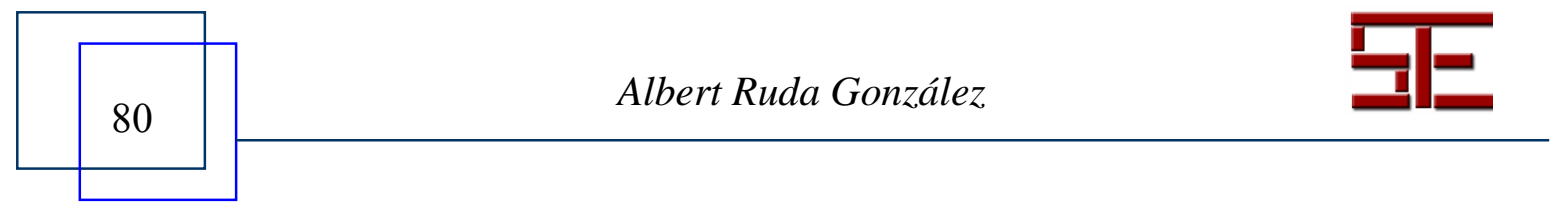




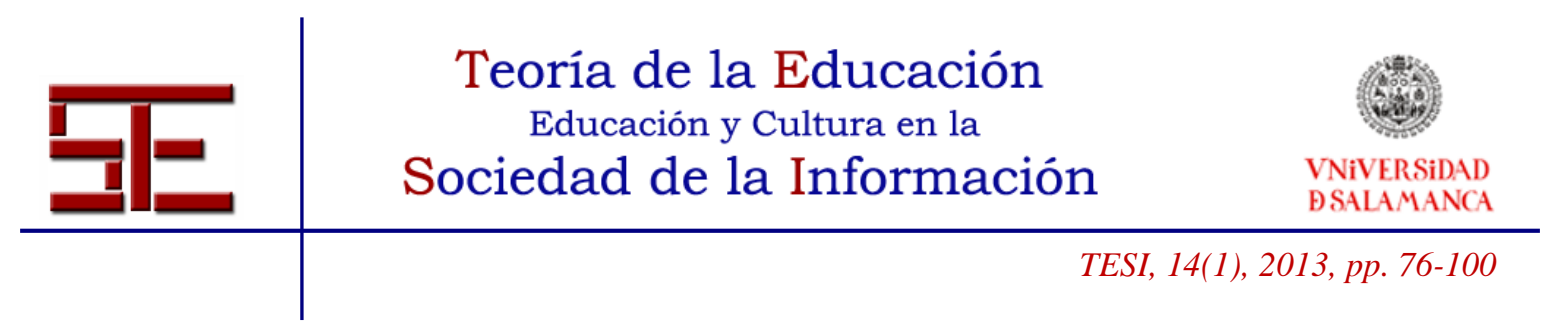

frustración (Kyung, 2007). Las herramientas tecnológicas para producir una colaboración entre los estudiantes ya existen, pero no siempre se introducen en la docencia de una forma sistemática (Brindley, Walter y Blaschke, 2009). Relacionado con el anterior, si no hay un espacio grupal de comunicación también esto puede hacer que los estudiantes se sientan frustrados, al privarles de la sensación de pertenecer a un grupo (Kyung, 2007). La literatura también alerta de que la efectividad del grupo disminuye cuando la comunicación es por ordenador, y que este mismo elemento acarrea un mayor tiempo de respuesta y menos satisfacción de los miembros (Baltes et al., 2002). Estos mismos autores conectan esa falta de satisfacción con la frustración estudiantil. La evaluación también es un elemento a tener en cuenta, sobre todo si el profesorado puntúa igual a todo el grupo. Entonces habrá un desincentivo para colaborar, ya que algunos estudiantes tenderán a trabajar menos, aspecto más acentuado en los ambientes virtuales (Kyung, 2007). También, la interacción con el ordenador lleva a menudo a la frustración cuando éste no hace lo que debería (Bessiere et al., 2004). La ansiedad y la frustración pueden deberse a que el estudiante carece de estrategias y habilidades adecuadas para la formación online (Borges, 2005), v.gr. porque nunca antes ha estudiado de ese modo. La creación de un sentimiento de cohesión grupal también es más importante en la enseñanza a distancia (Conrad, 2002).

En cuanto al aspecto de la falta de tiempo, aparece en la literatura como uno de los elementos que afectan a la satisfacción del estudiante en actividades colaborativas. Para empezar, el tiempo de dedicación es uno de los elementos de lo que hace el estudiante que puede generar frustración (Borges, 2005). Además, tener que interactuar con ordenadores a menudo requiere más tiempo (Baltes et al., 2002). A veces, necesita tanto tiempo para hacer su tarea que luego le falta para interactuar bien con los demás (Järvenoja y Järvelä, 2005). También la enseñanza online pide más disciplina por parte de los estudiantes (Allen y Seaman, 2007), lo que implícitamente puede acarrear problemas de escasez de tiempo si esa disciplina falta. También es importante que los profesores proporcionen feedback en un tiempo adecuado, de forma que se evite que el grupo se atasque o llegue a abandonar (Brindley et al., 2009). La falta de tiempo puede agravarse si los estudiantes no tienen una herramienta adecuada para comunicarse (An y Kim, 2007).

En efecto, como se va a ver más tarde al exponer la experiencia estudiada, para una parte significativa del grupo de alumnos, tener que trabajar con la wiki fue una simple pérdida de tiempo. Esto encaja con la literatura, que pone en conexión el sentimiento de pérdida

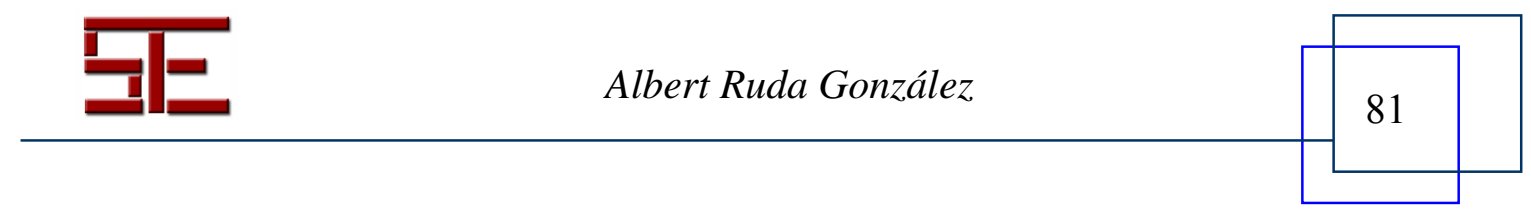




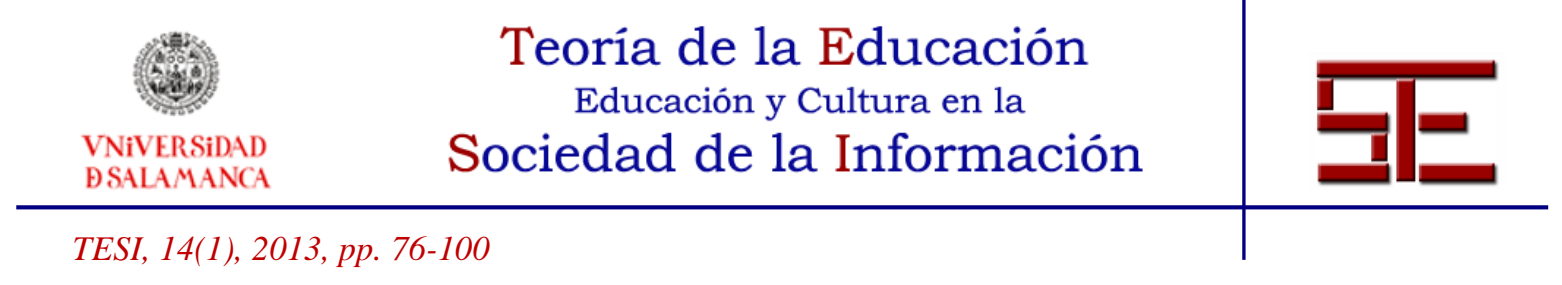

de tiempo para la interacción mediante ordenador con la pérdida de satisfacción por el estudiante (Bessiere et al., 2006). Sobre todo, ha contribuido a la percepción negativa de los estudiantes el hecho de tener que trabajar en la wiki de forma que el profesor pudiera hacer el seguimiento correspondiente. Probablemente el hecho de ser un grupo que se encuentra en clase cada día más o menos elimina o mitiga los problemas de comunicación virtual. A este respecto, la literatura señala que pueden surgir problemas de carga de trabajo debido al tiempo, que se pueden resolver mediante encuentros presenciales (Burdett y Hastie, 2009). De hecho, algunos grupos se reunían en persona para distribuirse el trabajo o incluso sentarse ante el ordenador para escribir conjuntamente. De aquí otra queja de los estudiantes, que cada uno de ellos tuviera que escribir por separado con un usuario propio.

En conexión con lo anterior, el hecho de que el grupo sea semipresencial (trabaja online en plan colaborativo, pero se encuentra en clase) puede mitigar otros problemas más graves en un entorno totalmente virtual. Por ejemplo, la confianza es fundamental en todo trabajo en grupo, y que ésta se mantenga durante toda la ejecución del trabajo. La falta de tiempo puede afectar a la construcción de la confianza necesaria entre los miembros del grupo (Goold, Craig y Coldwell, 2008). En cambio, los estudiantes del grupo en cuestión ya se conocían prácticamente en todos los casos. La confianza (o su carencia) podía construirse basándose en experiencias previas en otras asignaturas en algunos grupos. El entorno semipresencial hace que los estudiantes puedan interactuar cara a cara y por tanto esto podría facilitar la construcción de esa confianza. El hecho de que haya separaciones en el tiempo dificulta el trabajo del grupo, lo que puede llevar a una auténtica crisis del grupo mismo (Grinter, Herbsleb y Perry, 1999). En cambio, los malentendidos se pueden resolver más fácilmente con un contacto regular y la comunicación cara a cara (Hara y Kling, 1999). Esos problemas son más raros en el entorno presencial o semipresencial, al coincidir los estudiantes cada día en clase. Esto llama la atención sobre la necesidad de que en los cursos virtuales existan herramientas de socialización (Kreijns et al., 2004).

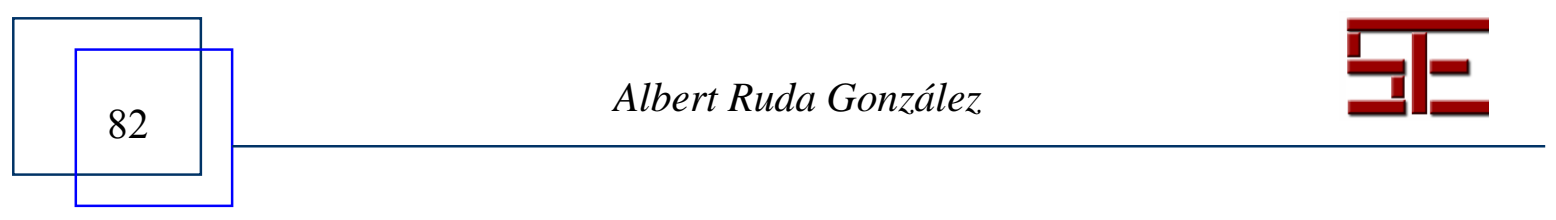




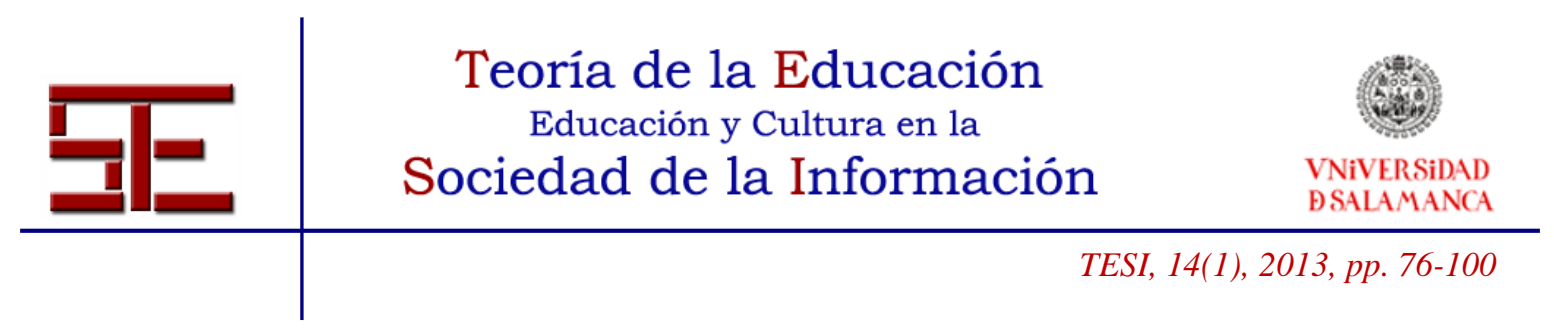

\section{3.- METODOLOGÍA DE LA INVESTIGACIÓN}

\section{1.- Aspectos metodológicos}

En lo tocante a la metodología, el estudio realizado se ha basado en primer lugar en un tratamiento de datos de tipo cuantitativo. Se trata de analizar la percepción de los estudiantes con respecto al aprendizaje cooperativo mediante wikis. En particular, se persigue identificar factores o motivos de frustración de los estudiantes y que éstos indiquen cuáles son los principales para ellos. Como es sabido, el método cuantitativo se caracteriza justamente porque los investigadores que lo eligen aspiran a adquirir una precisión y fiabilidad mediante prácticas estadísticas fiables y proceder de forma transparente y objetiva (Anderson y Kanuka, 2007). Por tanto, sí que es posible en teoría medir de forma cuantitativa la incidencia de determinados motivos sobre la frustración de los estudiantes.

De las diversas metodologías cuantitativas posibles, en este trabajo se ha aplicado la metodología ex-post-facto o no experimental, de hecho la más frecuente en la investigación educativa (Rodríguez y Valldeoriola, 2009). La elección se debe a que se analiza una experiencia real (no artificial), el investigador no puede controlar las variables y se trata en buena medida de un fenómeno ya acontecido. Conforme con esa metodología, se pretende dar respuesta a los interrogantes a tener en cuenta en toda investigación cuantitativa o empiricoanalítica (Creswell, 2004). Los participantes en el estudio son alumnos del Grado en Derecho, de $2^{\circ}$ curso, en modalidad semipresencial, en la Universitat de Girona. En concreto, los que hayan seguido la evaluación continua de la asignatura y participado en el aprendizaje colaborativo. Por tanto el grupo de sujetos ya está establecido. La selección tiene lugar automáticamente por el hecho de haberse matriculado en la asignatura. En cuanto a los instrumentos a utilizar, se han seleccionado por ser los que parecen más idóneos en relación con el tema y los objetivos de la investigación, y el hecho de escogerse una metodología empiricoanalítica. Al tratarse de encuestas anónimas, no pareció necesario solicitar permiso para utilizarlos. Sin embargo se advirtió de la posible utilización de los datos para estudios sobre investigación educativa o para la mejora docente. Fundamentalmente se ha utilizado la estadística descriptiva (observar y describir).

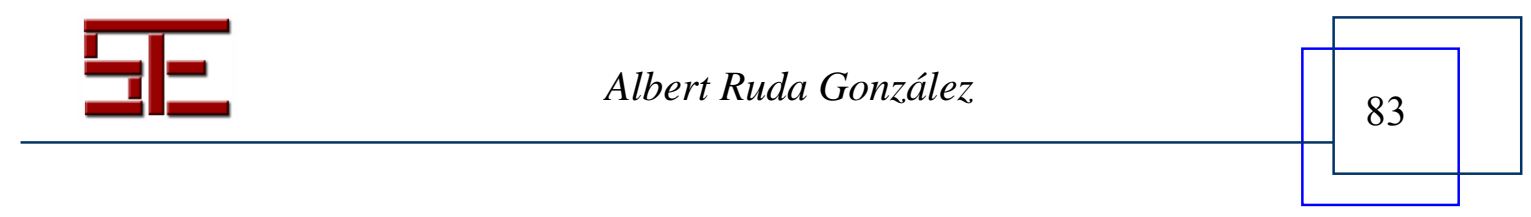




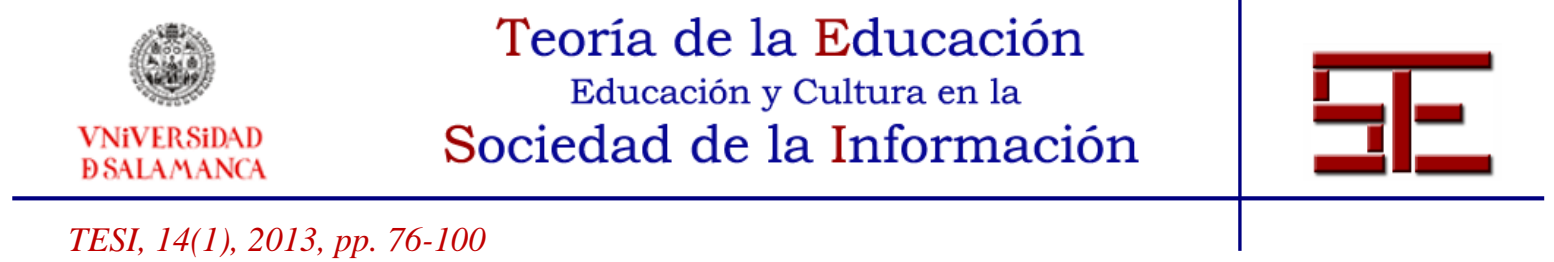

Ahora bien, como también es sabido, el enfoque cuantitativo puede combinarse con el cualitativo cuando ello ayuda a la obtención del objetivo de la investigación, e incluso ambos métodos se complementan recíprocamente (Neuman, 2006). En el caso que se plantea, los datos de tipo cualitativo pueden ayudar a entender cómo los estudiantes viven su frustración, si la hay, mientras que los datos cuantitativos pueden establecer una gradación entre la influencia de los motivos de esa frustración. Un enfoque combinado como el que aquí se sugiere fue utilizado, por ejemplo, en el estudio sobre las emociones en el aprendizaje online de Rebollo, García, Barragán, Buzón, y Vega (2008).

De acuerdo con lo que se ha apuntado más arriba, los instrumentos tienen que permitir identificar, cuantificar y entender los motivos de frustración de los estudiantes. A tal efecto, se han tomado en cuenta el registro anecdótico o de observaciones, la observación en comunidades virtuales y un cuestionario. En cuanto a lo primero, se recogieron comunicaciones de los alumnos al profesor, en las que se manifestase un sentimiento de frustración, ya sea dentro o fuera del aula. En particular, cabe referirse a una charla con el delegado de uno de los grupos, justamente para expresar la frustración de sus compañeros y el motivo o motivos, o bien a los sentimientos expresados por ellos mismos en las sesiones de tutoría presencial fuera de las horas de clase.

Respecto de la observación anecdótica, se han tomado en consideración las evidencias que los alumnos aportan mediante sus comunicaciones en el espacio de trabajo virtual de la asignatura. Se trata de un espacio de Moodle en el cual los alumnos se integran como una especie de comunidad virtual, que incluye foros y listas de distribución. En este caso, la interacción entre los estudiantes se produce en un mundo diferente de la presencialidad, en un "no lugar" (Suárez, 2010). La observación busca indicadores de la existencia de frustración estudiantil. Desde un punto de vista psicológico, en tanto que emoción, la frustración puede reflejarse en: a) lenguaje expresivo y evaluativo (e.g., expresiones faciales y autoinformes), b) cambios fisiológicos y c) secuencias conductuales, tales como patrones de evitación o rendimientos empobrecidos (Kamenetzky et al., 2009). De estos aspectos, en el contexto de esta investigación parecen observables sólo los elementos a) $\mathrm{y} \mathrm{c)}$.

Esa observación se ha complementado con la toma en consideración de los textos elaborados cooperativamente (como señalan Anderson y Kanuka, 2007). Por tanto se llevó a cabo una actividad de rastreo de las actividades en línea, análisis de transcripciones de textos de actividades sociales o de aprendizaje (de acuerdo con lo que apuntan

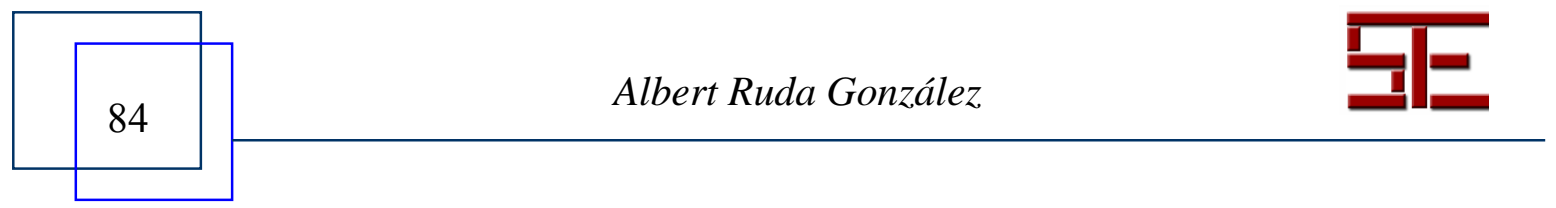




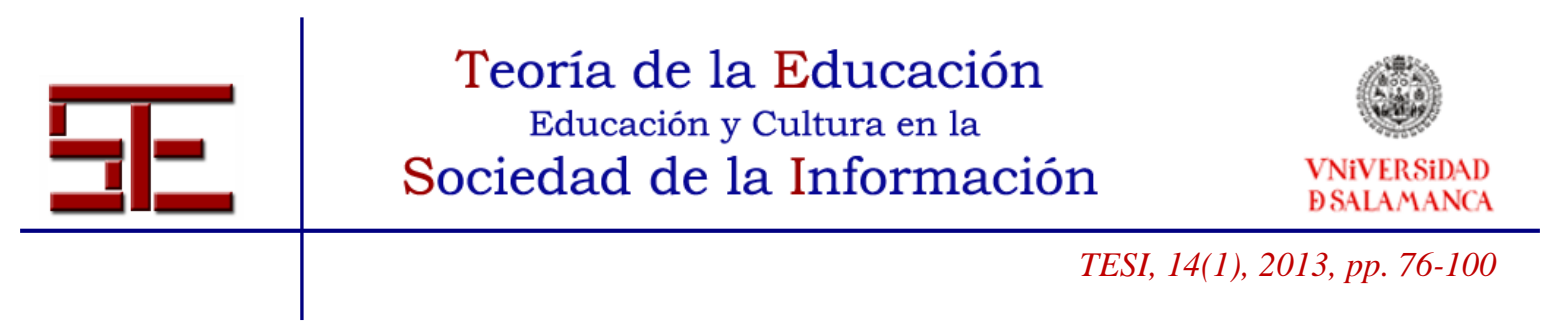

Anderson y Kanuka, 2007). Ello incluye los foros de Moodle y los espacios de discusión de Wikispaces. Para ello ha sido precisa una tarea notable de construcción de significados, ya que había que dotar de sentido a expresiones lingüísticas empleadas por los alumnos del curso en cuestión, como signos de admiración o uso de mayúsculas, inter alia.

Con todo, el principal elemento de juicio aquí expuesto es un cuestionario que se propuso a modo de encuesta a los estudiantes que habían participado en la actividad de aprendizaje colaborativo. Se trata de una herramienta de recogida de datos muy conveniente, habida cuenta del elevado número de alumnos implicados $\mathrm{y}$, por ende, de la práctica imposibilidad de hacer entrevistas a todos ellos. El cuestionario se utiliza en un único momento, al final de la experiencia base. Sólo en ese momento el alumno estará en condiciones de afirmar si ha sentido frustración y, en caso afirmativo, cuáles son los motivos. Así pues, una vez realizados los trabajos, se hizo una encuesta a los alumnos que los habían presentado. La encuesta fue de formato electrónico y con carácter voluntario. Se escoge ese formato electrónico dado que presenta numerosas ventajas respecto de su realización en papel (Bryman, 2008). En particular, después de estudiar diversas opciones se escogió el cuestionario mediante Google Docs (Google Drive).

En la encuesta se incluye una parte de preguntas generales sobre la actividad, y otras específicas sobre la motivación y la frustración de los estudiantes. Algunas de las preguntas se centran en aspectos que el profesor ya ha ido observando durante la ejecución de la actividad y anotado en el registro de anécdotas. Obviamente, puede haber aspectos que pasen desapercibidos al profesor. De hecho, en el diseño de la encuesta pueden influir ciertos supuestos que permanecen ocultos o de los que no siempre se es consciente (Sánchez Carrión, 2012). Como es sabido, generalmente solo se ve aquello que se busca, de modo que parece conveniente dejar alguna pregunta abierta para que los alumnos puedan aportar cosas en las que el profesor no haya reparado. Así se ha hecho, mediante dos preguntas. Una, destinada a preguntar al alumno cómo se ha sentido al realizar la experiencia. La otra, al final del cuestionario, para que pueda añadir lo que considere oportuno.

Los alumnos invitados a responder la encuesta son todos los que han participado en el aprendizaje colaborativo. La encuesta se hace mediante un aviso en el tablero de anuncios de la asignatura. En el espacio virtual se incluye una explicación sobre la encuesta y su funcionamiento. Ahora bien, existe un cierto riesgo de que algunos no respondan

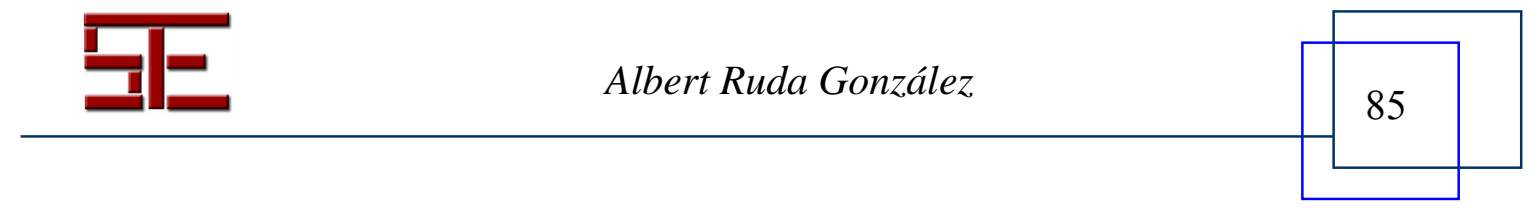




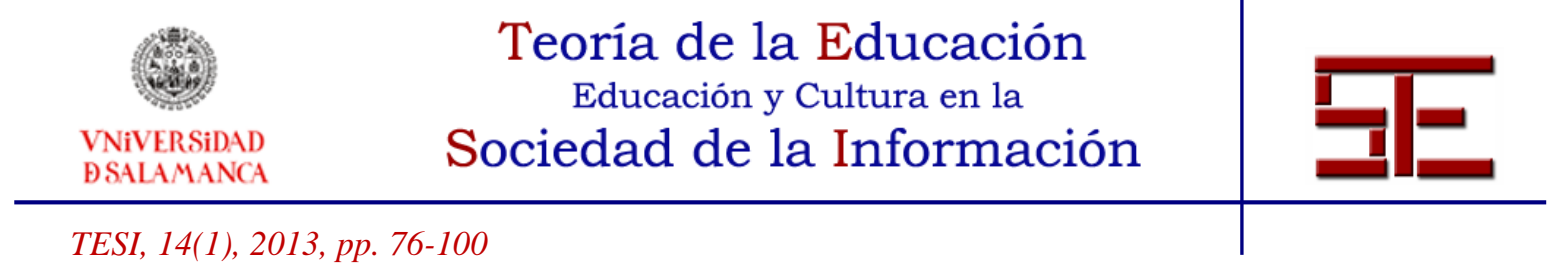

(nonresponse), sea porque no han concluido el trabajo, han abandonado la asignatura, no han tenido tiempo, u otro motivo. Como dice Flick (2006), el entorno online limita a los entrevistados de los relevantes a los accesibles. Por ello se complementará esta información con la ya referida.

A los efectos de planificar la encuesta, hay que tener en cuenta que se trata de dos grupos de alumnos: Grupo A (mañanas) y Grupo B (tardes). El número de matriculados es de 109 y 87, respectivamente. Descontando las anulaciones de matrícula, quedan en $101 \mathrm{y}$ 75. De ese total, sólo una parte está participando en el trabajo colaborativo, ya que algunos alumnos han dejado la asignatura sin haber anulado la matrícula, o han suspendido ya la asignatura por copia en un examen parcial (una persona en el grupo B). Por ende, el número de encuestados ya desde el principio se previó que fuese menor. En cualquier caso, se trata de una encuesta realizada en línea. Para ello se tienen en cuenta las características de este tipo de encuestas y potenciales problemas (siguiendo a Couper, 2000).

El cuestionario o encuesta se basa en buena medida en los resultados de la investigación precedente y especialmente en el estudio fundamental de Capdeferro y Romero (2012). También se ha tenido en cuenta el estudio sobre la satisfacción de los estudiantes en el blended learning de Melton, Graf y Chopak-Foss (2009). En cuanto a las preguntas, se toman en consideración los elementos clave del trabajo colaborativo en entornos virtuales, como son la coordinación del proceso, la organización y planificación de las tareas, la gestión de la información, la comunicación e interacción, y la ética de trabajo personal (Guitert y Giménez, 2000). Se tiene en consideración especialmente que la frustración afecta a la motivación del estudiante. Por tanto, para encarar la frustración es fundamental también comprender qué factores afectan a la motivación. Sobre todo, hay que partir de la premisa de que la falta de motivación no es responsabilidad del alumno, sino que en ella influyen muchos elementos relativos a su interacción consigo mismo y con sus iguales, con sus profesores y con las experiencias y contenidos de aprendizaje brindados por éstos, y de la interacción de su historia de éxitos y fracasos y de las expectativas sugeridas por el tipo de enseñanza (Echeita, 2003).

Los motivos de frustración posibles que se han incluido en el cuestionario responden a lo que se ha apuntado más arriba sobre factores que pueden producirla. Se han incluido en ese bloque sub-preguntas sobre aspectos diversos: la actuación del profesor, los aspectos técnicos, la relación entre los estudiantes o el aspecto puramente personal del encuestado.

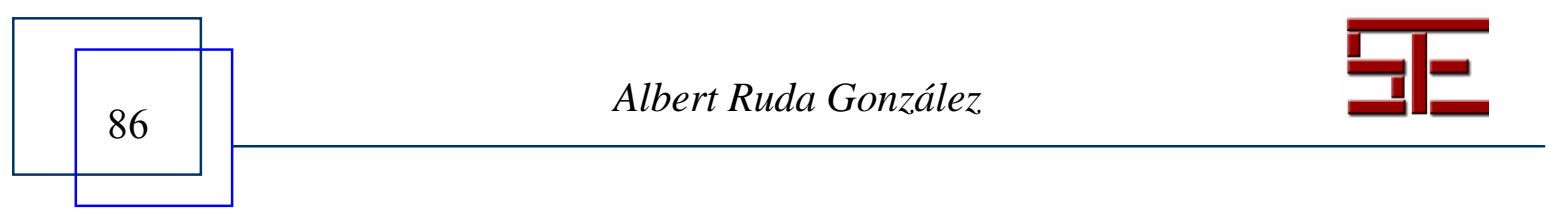




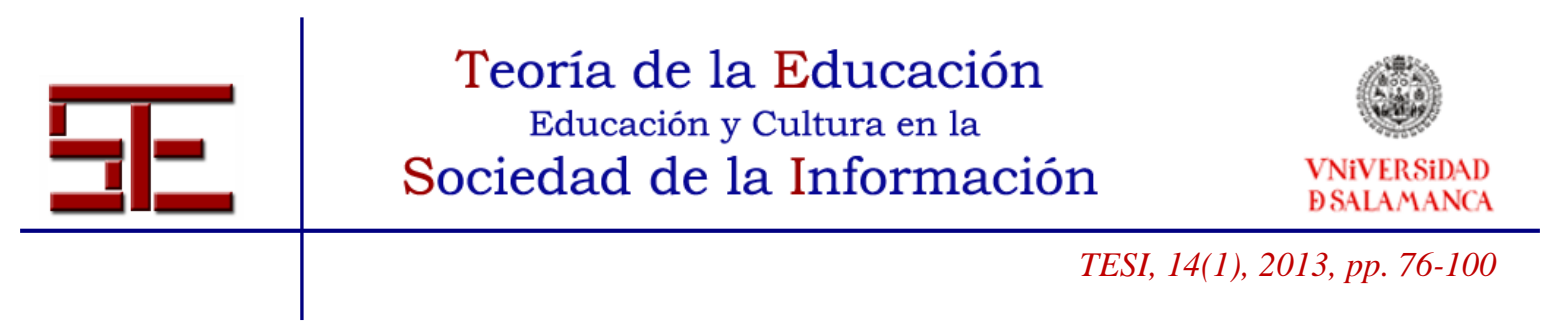

Para acabar con el aspecto metodológico, cabe añadir que durante la realización del estudio se realizó una sesión de seguimiento presencial, aparte del seguimiento realizado a través del espacio virtual de la asignatura y el correo electrónico y tutorías. En dicha sesión presencial el profesor interrogó a los alumnos sobre su estado de ánimo. Se trata de una especie de grupo focal (focus group) pero con más alumnos, ya que el número es más elevado que el habitual en esa técnica (que suele ser de unos 10 aprox.). La impresión que obtiene el profesor-investigador puede ayudar a la interpretación que se tiene que hacer de los resultados de los demás instrumentos. En este sentido, el registro anecdótico puede interpretarse como corresponda, pero puede quedar la duda de si realmente se corresponde con las emociones que querían expresar los alumnos. En ese sentido, hay que tener en cuenta el aspecto de la llamada "validación del respondente" (respondent validation) (Bryman, 2008). La entrevista grupal puede servir para paliar esa deficiencia. A lo anterior se podría añadir la sesión de presentación pública de los trabajos colaborativos. Sin embargo, al tratarse más bien de un conjunto de exposiciones, y por razones de tiempo, no se realizó una segunda entrevista grupal.

\section{2.- Escenario de la investigación}

La investigación llevada a cabo toma como punto de partida la actividad práctica realizada por estudiantes de una asignatura de Derecho (Derecho de la propiedad y Derechos reales) en la Universidad de Girona durante el segundo semestre del curso 2011-2012. Se trata de una asignatura impartida, como el resto del Grado en Derecho, presencialmente. Sin embargo, una parte de la nota final depende de la realización de actividades de tipo práctico. En concreto, la realización de un trabajo de investigación en grupo (Sharan y Sharan, 2004) supone el 30\% de la nota final. Los estudiantes realizan el trabajo fuera del aula y el profesorado les propone el empleo de wikis en la plataforma Wikispaces como herramienta de trabajo colaborativo basado en ordenador (CSCL). La Universidad donde se realiza la actividad no dispone de espacio propio en Wikispaces, de modo que los estudiantes aprenden por su cuenta el funcionamiento de las wikis, las crean y editan.

La definición de la actividad por parte del profesorado es relativamente abierta. Se propone un tema marco (la propiedad intelectual) a principio de curso y cada grupo decide cómo quiere enfocarlo, de modo que se cumple la recomendación de que la actividad colaborativa sea abierta (Úriz, 1999). Los grupos se forman libremente por parte de los estudiantes y duran todo el semestre. En el aula se hacen dos sesiones de seguimiento con

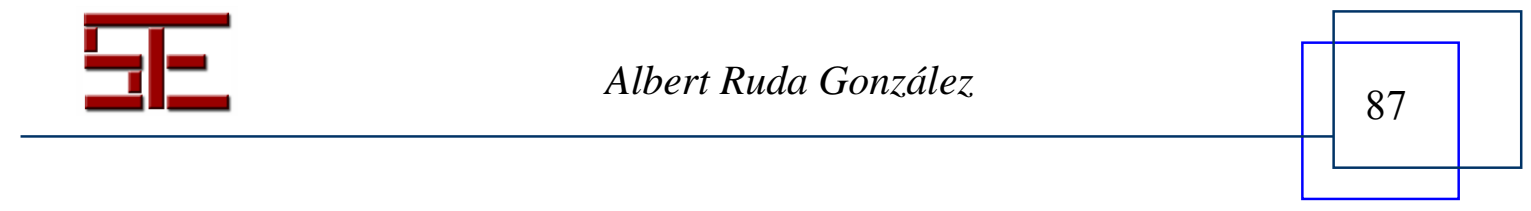




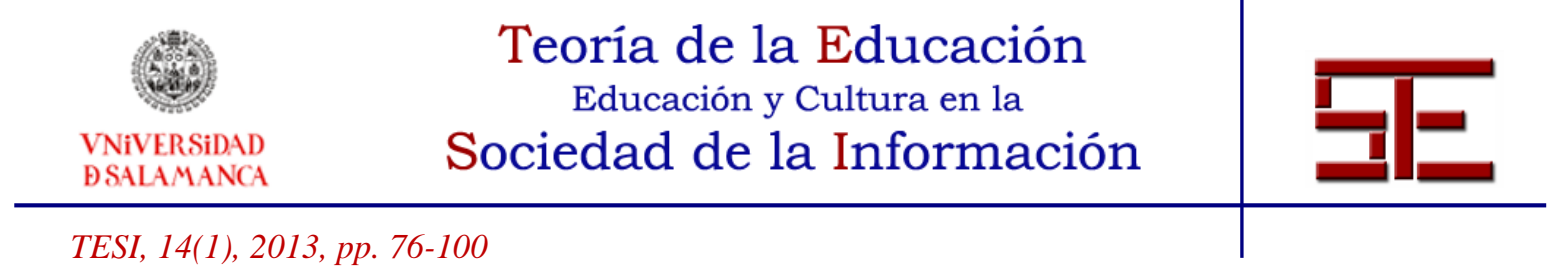

el profesorado, una a medio curso y otra al final, días antes de la presentación pública de los trabajos.

\section{3.- Recogida y análisis de datos}

Acabada la actividad, se realiza una encuesta a los estudiantes para evaluar su satisfacción, especialmente con el empleo de las wikis. Uno de los aspectos principales de la encuesta fue cómo se habían sentido los estudiantes, si se habían sentido frustrados $\mathrm{y}$, en caso afirmativo, por qué. Se sugirieron una serie de posibles factores de frustración que los encuestados podían puntuar. De 176 matriculados respondieron $76(n=76)$, o sea el $43,18 \%$ del total. El promedio de la edad de los alumnos que han respondido es de 23,02 años. Por sexos, hay un número bastante mayor de mujeres (52, es decir un $68,42 \%$ de los respondientes) en relación con los hombres (24, equivalente al 31,57\% restante).

\section{4.- RESULTADOS}

Una gran parte de los respondientes declaran que sí han realizado trabajos colaborativos con anterioridad (53), esto es casi el 70\%, frente a los que no (23, esto es, el 30,26\%). El resultado parece sorprendente, ya que prima facie muy pocos profesores utilizan ese método en su docencia en Derecho (aunque habría que tener en cuenta la formación preuniversitaria). La mayoría declaran que han trabajado anteriormente con otras tecnologías: redes sociales $(32,89 \%)$, blogs $(18,42 \%)$, foros $(5,26 \%)$, u otras tecnologías $(9,21 \%)$, o ninguna $(34,21 \%)$. Muy pocos estudiantes han trabajado con wikis con anterioridad $(4,1 \%)$.

Con respecto a la valoración de la experiencia con wikis, a la pregunta de si les gustaría hacer más trabajos colaborativos en el futuro, 31 responden que sí $(40,78 \%)$, y el resto que no $(59,21 \%)$. Por tanto, mayoritariamente no les gustaría repetir la experiencia. En general, tampoco les gusta trabajar en grupo, ya que puntúan este tipo de trabajo con un 4,76 sobre 10 de media. Esta puntuación contrasta con el resultado obtenido a la pregunta siguiente, relativa a si los encuestados creen que les serían útiles estrategias para trabajar en grupo de forma virtual. La mayoría piensa que sí, con 40 respuestas $(52,63 \%)$, mientras que 25 creen que no $(32,89 \%)$ y 11 contestan NS/NC $(14,47 \%)$. La gran mayoría de los

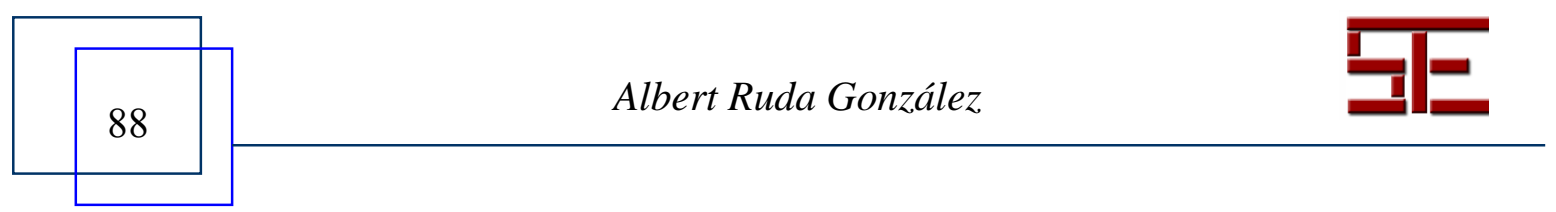




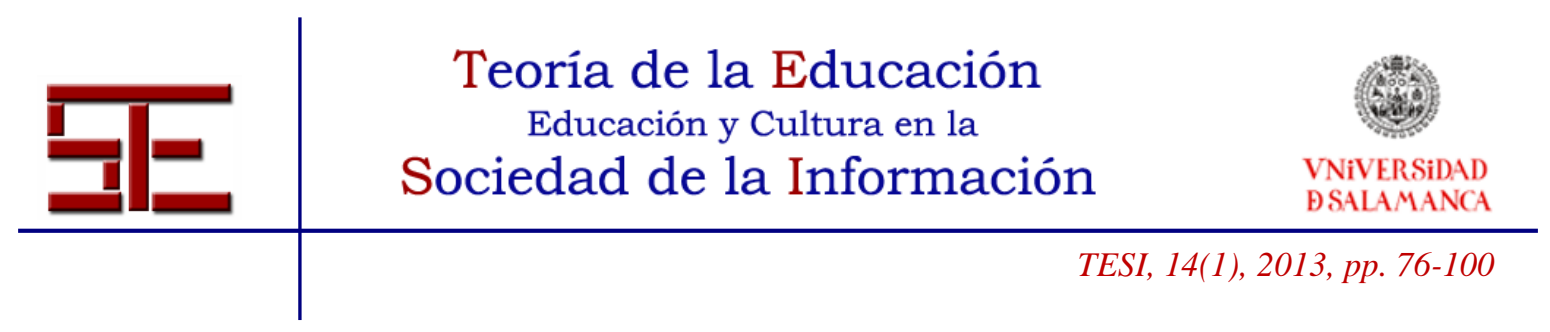

encuestados declara que trabaja mejor individualmente $(77,63 \%)$ que en grupo $(15,78 \%$; el NS/NC obtiene el 6,57\%).

Ya en relación con los trabajos colaborativos, a la pregunta de si los alumnos los prefieren a las clases magistrales la respuesta es negativa. En una escala de 0 a 10, responden de promedio un 3,09, por tanto, bastante por debajo del aprobado.

Si se centra ahora la atención en las preguntas específicas sobre la frustración, para empezar hay que referirse a los comentarios escritos de los alumnos. Se centran en: a) falta de tiempo; b) estrés; c) falta de información; d) las propias características de la herramienta utilizada; e) la falta de colaboración de los compañeros del grupo; f) sentirse poco recompensados en términos de nota, frente a los estudiantes menos colaboradores; g) la imposibilidad de llegar a acuerdos; h) falta de aprendizaje; i) falta de comunicación; j) preferencia por el trabajo individual, y k) falta de interés por el tema del trabajo. Una pregunta fundamental es si los alumnos consideran que han conseguido sus objetivos con su trabajo. En una escala de 0 a 10, el resultado de media es 6 . A contrario, sugiere que un poco menos de la mitad no consideran haberlos logrado, y es en ese porcentaje de alumnos en los que más podría haberse notado la frustración. A la pregunta específica de si se han sentido frustrados en algún momento, por qué motivo ha sido, solo responden 27 de los 76 alumnos (el 35,52\%). Muchos de ellos (8 en concreto) se limitan a contestar "sí", sin ningún otro comentario (30\% de los que han respondido esta pregunta). Otros señalan como motivo a la falta de tiempo (4), los compañeros (9), la manejabilidad de los wikis (5), haberse sentido solo (1), no haber entendido qué se pedía (1), por "todo en general" (1), y uno simplemente señala que "no" se ha sentido frustrado. En relación con el ítem numéricamente más importante, 8 de los 9 señalan que la frustración deriva de la falta de colaboración o compromiso, y uno a una falta de entendimiento sobre el reparto de la tarea entre ellos. Por ende, la relación con los compañeros aparece como el motivo principal de frustración (véase la tabla siguiente).

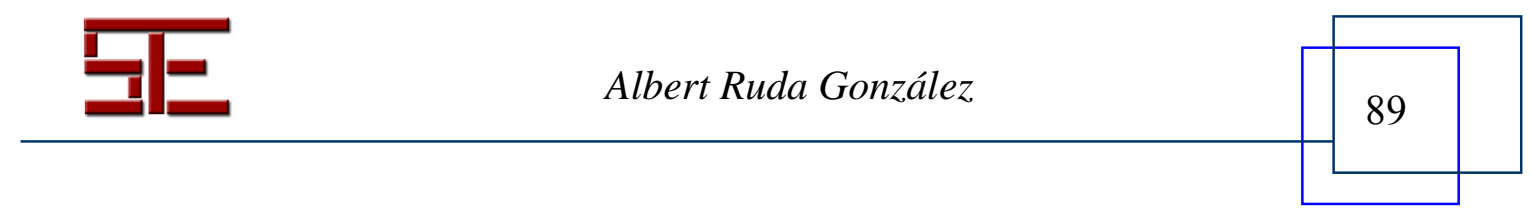




\begin{tabular}{cc} 
Teoria de la Educación \\
Educación y Cultura en la \\
$\begin{array}{c}\text { VNiVERSADAD } \\
\text { BSALAMANCA }\end{array}$ \\
\hline TESI, 14(1), 2013, pp. 76-100
\end{tabular}

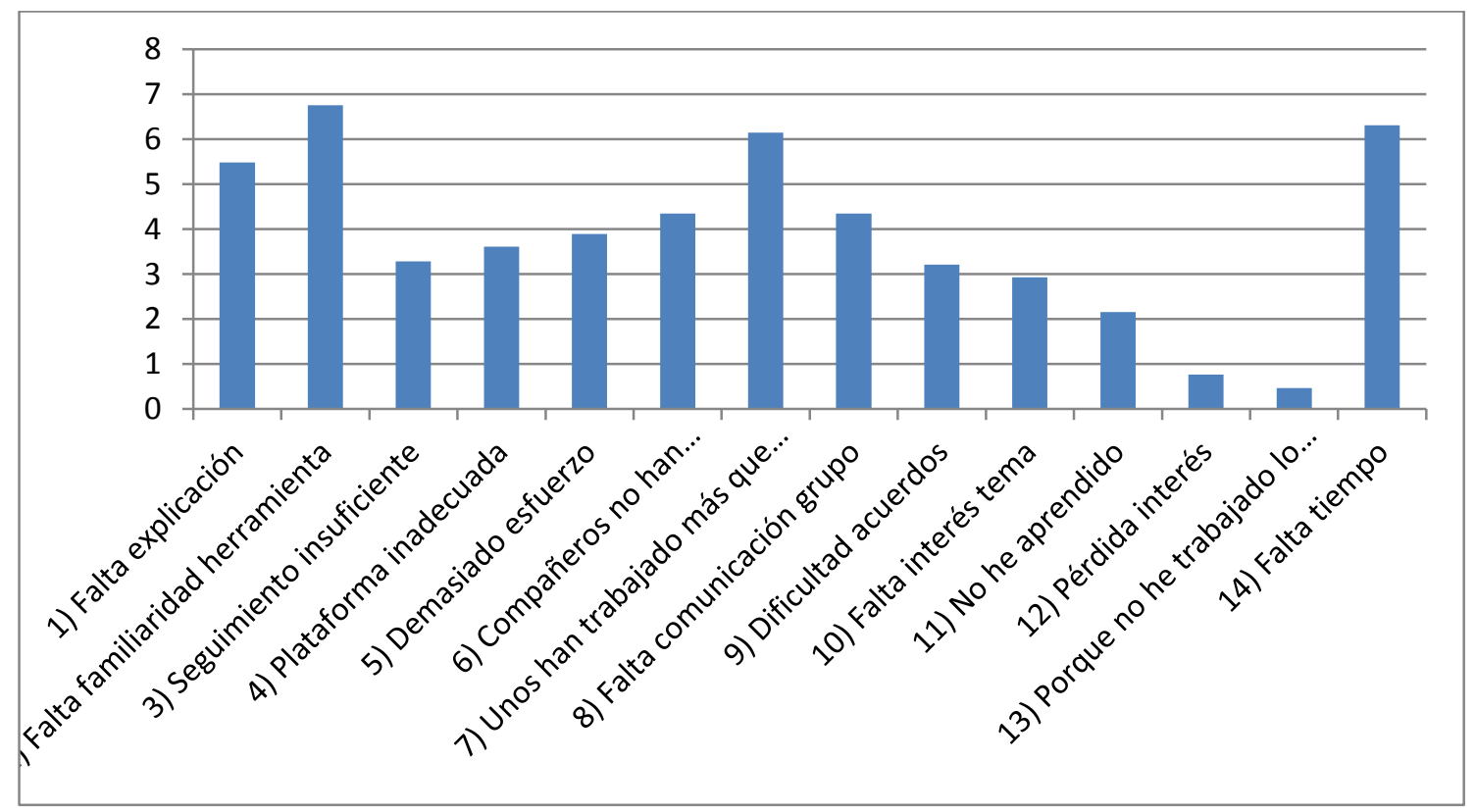

Tabla 1. Motivos de frustración en los estudiantes

En cuanto a las razones de la frustración, la más señalada por los estudiantes es la falta de familiaridad con la herramienta wiki (puntuada de media con un 6,75 sobre 10). Le siguen empatados la desigualdad en la carga de trabajo entre miembros del grupo $(6,15$ sobre 10), y la falta de tiempo (6,31 sobre 10). Muy cerca, sigue la falta de explicación por parte del profesor $(5,48$ sobre 10$)$, que posiblemente quepa relacionar con el motivo señalado en primer lugar (pues más explicación hubiese contribuido seguramente a hacer la herramienta más familiar al alumno).

\section{5.- DISCUSIÓN}

Como se desprende de los comentarios de los alumnos, la valoración que han hecho de la actividad colaborativa mediante la encuesta parece predominantemente negativa. Así lo sugiere, en particular, el deseo de no repetir la actividad. Los motivos son diversos. Los estudiantes prefieren trabajar de modo individual. En tanto que el aprendizaje colaborativo es una subclase del trabajo en grupo (Monereo y Duran, 2001), ello ya da una pista sobre su relativa poca disposición para utilizar ese método de aprendizaje. Si

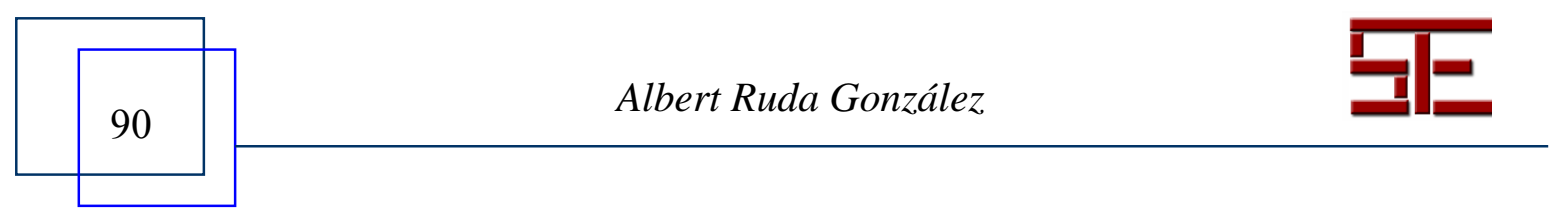




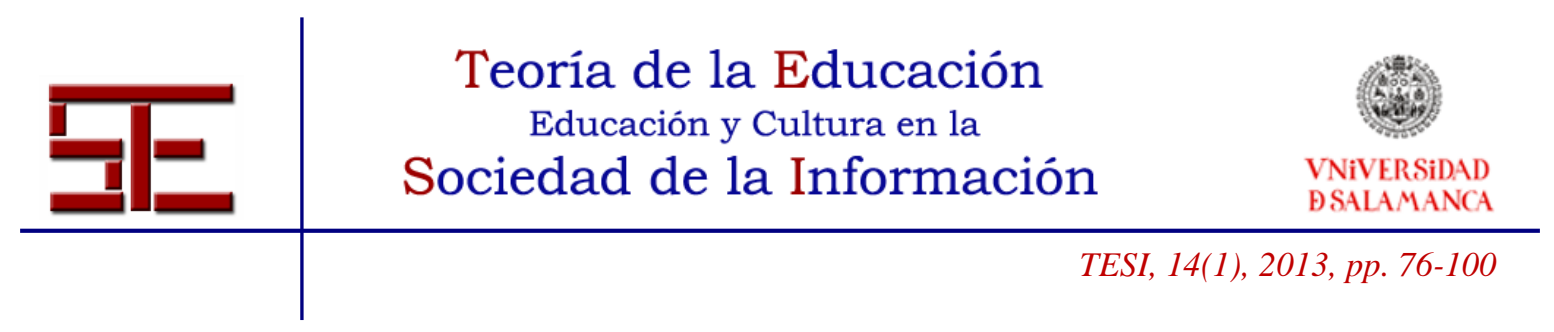

tienen que hacerlo, prefieren el trabajo en grupo no colaborativo, sin la herramienta wiki. Si esta se utiliza, proponen que sea de uso no obligatorio sino optativo.

En cuanto al tipo de docencia, prefieren las clases magistrales al trabajo colaborativo. Este punto parece abierto a la discusión ya que, como señala la literatura, el aprendizaje colaborativo no necesariamente debe desplazar al aprendizaje competitivo, sino que los profesores deben saber cuándo utilizar cada uno de ellos (Monereo y Duran, 2001). Además, es posible que una experiencia no del todo satisfactoria haga decantar a los alumnos hacia la clase magistral cuando en realidad en su conjunto el trabajo colaborativo puede ser más indicado para un tipo de aprendizaje o situación. Por ejemplo, algunos alumnos han comentado que se sentían desorientados porque no sabían qué se esperaba de ellos. Sin embargo, el reproche es muy inconcreto. De hecho, el mismo planteamiento de una actividad colaborativa puede que ser abierto (parecidamente, Nuutila et al., 2008). Por ende, ese aspecto negativo puede ser difícil de corregir sin desvirtuar la actividad.

Gran parte de los reproches de los estudiantes se deben al empleo de la wiki, lo que es coherente con otros datos recopilados (comunicaciones con el profesor). Los alumnos casi en su totalidad no conocen la herramienta y se quejan en la encuesta del tiempo que tienen que dedicar a aprender cómo funciona. En un contexto de aprendizaje en el que las asignaturas prácticamente bombardean a los estudiantes con actividades de todo tipo, un número considerable de estudiantes señala que esas horas son tiempo perdido. Los estudiantes se quejaron por no haber sido informados lo suficiente sobre el funcionamiento de las wikis. Este hecho refuerza la idea de que el dominio de la herramienta tecnológica es un requisito para el trabajo colaborativo virtual (Guitert y Giménez, 2000). Además, la frustración al inicio del uso de un recurso nuevo es un problema recurrente en trabajos colaborativos (por ejemplo, Naidu y Oliver, 1999). En general, esa frustración desaparece en un estadio más avanzado de la actividad, como de hecho sugieren algunos comentarios de los estudiantes.

Algunos estudiantes critican que el empleo de dicha herramienta sea obligatorio. En una sesión de seguimiento presencial, una alumna llega a preguntar si el empleo de la wiki es un fin en sí mismo, o bien una herramienta, un medio para conseguir un objetivo. Está claro que el web es un simple medio, un sistema diferente de interrelación y de trabajo, pero no un fin per se (Duart y Sangrà, 2000). Pero los alumnos pueden tener la sensación de que el profesor se empecina y con una herramienta ello limita su libertad de organizar

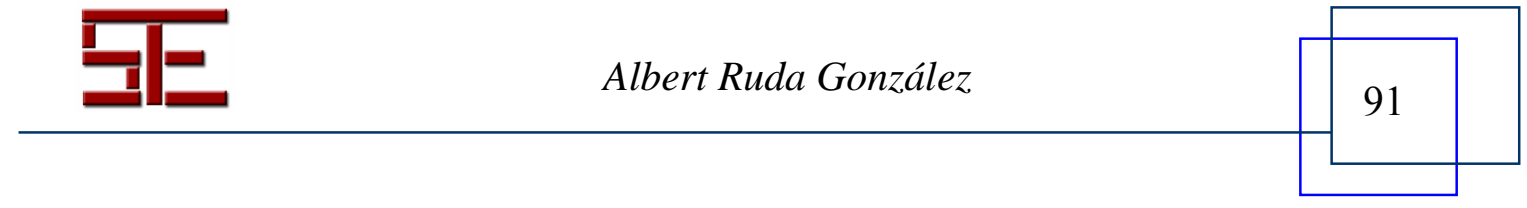




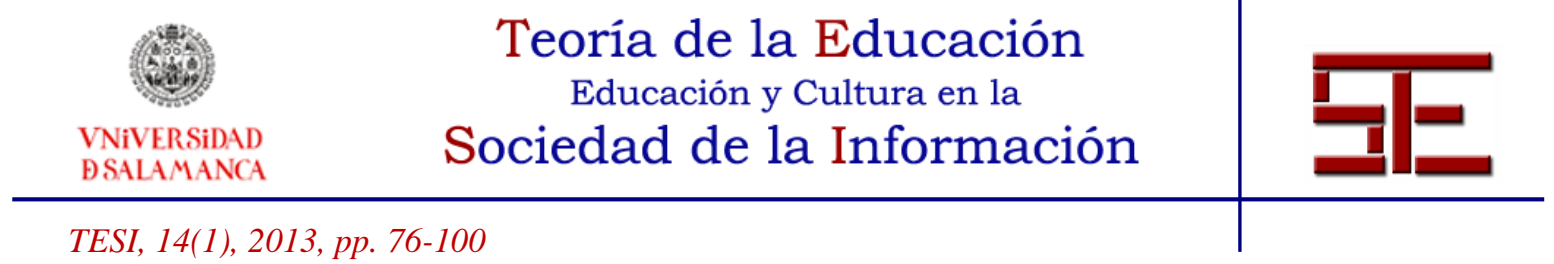

el trabajo. O bien, pueden pensar que aprender a utilizar la wiki no les va a servir en el futuro (como parece sugerir un estudiante en la encuesta; véase parecidamente Liu et al., 2011). Por ello, una muestra de flexibilidad, como es haber permitido el empleo de herramientas alternativas (como el blog), fue recibida con los brazos abiertos por los estudiantes.

Extrañamente, también se ha percibido como un inconveniente el hecho de que se utilice un recurso en línea. En unos estudios fundamentalmente presenciales, los alumnos no están acostumbrados a trabajar sin verse cara a cara (face-to-face). La crítica sorprende, ya que una de las condiciones que permiten la colaboración del grupo es justamente la interacción cara a cara, es decir, la maximización de las oportunidades de interacción entre los estudiantes (Monereo y Duran, 2001).

En cambio, prima facie parece coherente con lo anterior que otro de los ítems que producen más malestar sea la contribución inequitativa al trabajo del grupo. Como han señalado diversos autores (particularmente, Johnson, Johnson y Holubec, 1999), la interdependencia positiva es la clave para la cooperación efectiva. Si un miembro del grupo no coopera, esa interdependencia se ve afectada. Mas, si bien se mira, uno se podría interrogar si esto es realmente así. Si en un grupo colaborativo unos alumnos están trabajando más, hasta el punto de que uno haga el trabajo de los demás, algo falla, no en la distribución del trabajo, sino posiblemente en el mismo diseño de la actividad. Si el trabajo es realmente colaborativo, los alumnos dependen los unos de los otros, y por ende no es posible ex definitione que uno haga la parte de los demás (insiste en este extremo Fortner, 2002). Si la carga inequitativa produce frustración, ésta es un indicador de ese desequilibrio, y éste, de la mala configuración de la actividad por parte del profesor. Por tanto, cabe plantear la hipótesis de que si se produce frustración en el trabajo llamado "colaborativo", o bien nos encontramos ante un trabajo que en realidad es de otro tipo (un trabajo en grupo tradicional) o bien la actividad es realmente colaborativa y, por tanto, la frustración se debe a otro motivo distinto. La razón es que un trabajo en el que uno salva al grupo no hay interdependencia, sino solo dependencia del salvado respecto del salvador. Por tanto, falta un requisito sine qua non del trabajo colaborativo.

Aparte, ya se ha visto que la falta de tiempo es otro de los motivos de frustración más importantes. Como señala la literatura, "que el aprendizaje cooperativo sea efectivo depende de la cantidad de tiempo que los alumnos trabajen en equipo y de la calidad de ese trabajo" (Pujolàs, 2008). Esa falta de tiempo se puede deber a una mala planificación

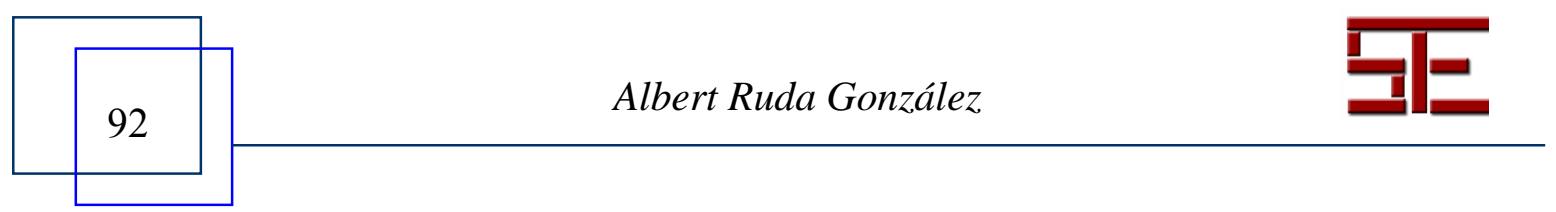




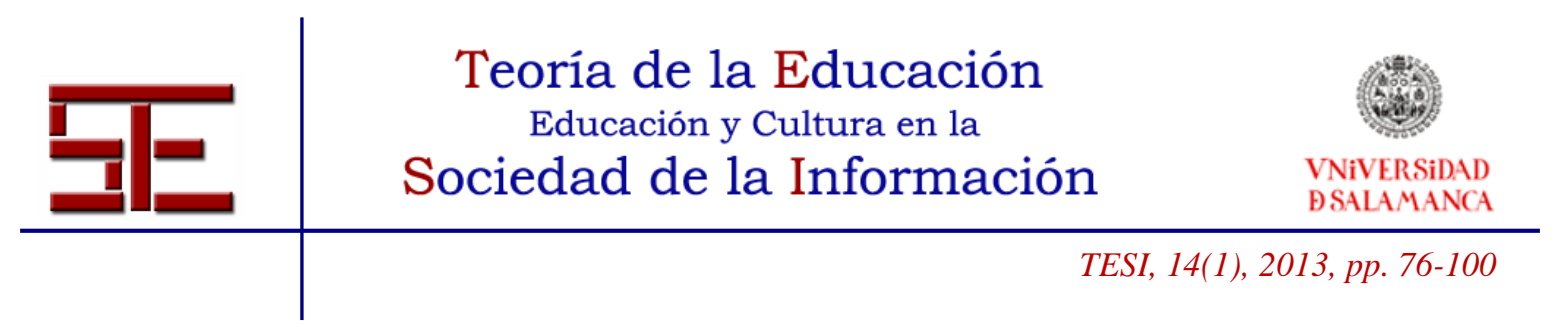

de la actividad por el profesor, a una mala ejecución por los alumnos, a un exceso de trabajo por parte de éstos (teniendo en cuenta las demás asignaturas), o incluso al tamaño de los grupos (pues se ha sugerido que a menos tiempo disponible, menores deben ser aquéllos, véase Ovejero, 1990). No obstante, puede descartarse este elemento, ya que los grupos eran de 3 personas y por tanto no excesivamente grandes, sino medianos tirando a pequeños. Solo un alumno criticó que los grupos deberían haber sido de dos y no de tres miembros.

Además, el hallazgo de que la falta de tiempo es uno de los principales motivos de frustración es coherente con la literatura. En particular, en ella se pone de relieve que en el aprendizaje cooperativo se tiende a atribuir el fracaso a la dificultad de la tarea, la mala suerte y la falta de esfuerzo de los miembros del grupo (Ovejero, 1990). Como se ha visto, ningún alumno alude a la mala suerte en sus comentarios, y tampoco nadie dice que el trabajo fuese demasiado difícil. A diferencia de lo que ha sucedido en otros casos, no se ha planteado que la frustración derivase de un excesivo desequilibrio entre las habilidades de los estudiantes en la clase (cf. Andrews, 1998). En cambio, atribuir el malestar generado a la falta de tiempo puede ser una forma indirecta de aludir a la dificultad de una tarea (pues una tarea más difícil, y lo era aprender el funcionamiento de la wiki) generalmente requerirá más tiempo. También se ha visto que los alumnos culpan de su frustración a otros compañeros suyos, lo cual es nuevamente coherente con la literatura.

Lo que no es coherente es que la falta de tiempo tenga tanto peso como factor que motiva la frustración. En un estudio anterior sobre este tema, estaba muy por debajo del desequilibrio entre el trabajo de los miembros del grupo (Capdeferro y Romero, 2012). En cambio, en el presente estudio se ha visto que prácticamente tienen el mismo significado en términos estadísticos, mientras que en los comentarios incluso se da más peso a la falta de tiempo que al otro aspecto. Este trabajo sugiere, pues, que la falta de tiempo es tan importante (o más) como la falta de dedicación de los compañeros, en contraste con el estudio citado. En cambio, de nuevo frente a este estudio, no se aprecian dificultades de comunicación significativas (posiblemente debido a que los estudiantes se mueven en un entorno presencial y no solo virtual, a diferencia del entorno en que se hizo el estudio de Capdeferro y Romero (2012).

En el fiel positivo de la balanza hay que subrayar el hecho de que los alumnos consideren mayoritariamente que han aprendido. O mejor dicho que no se han frustrado por el hecho

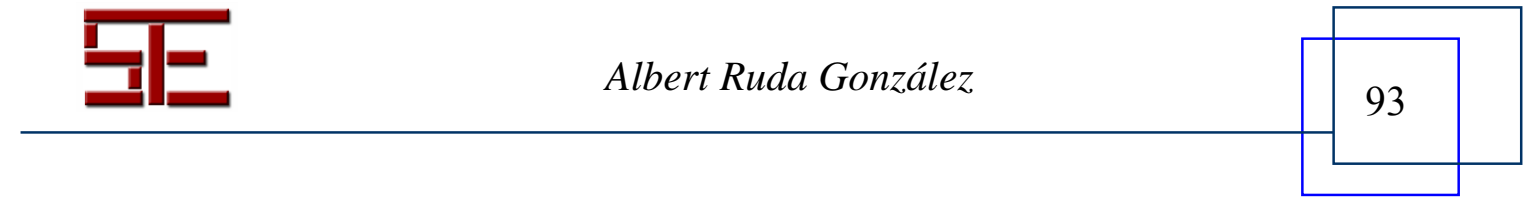




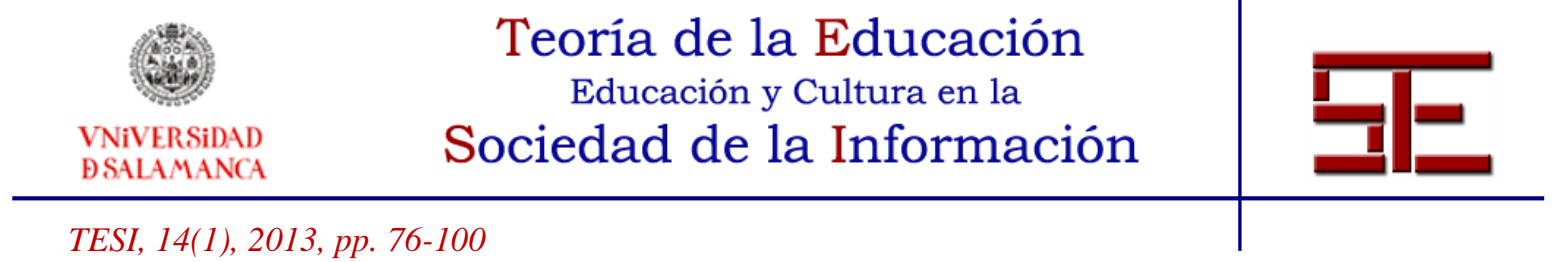

de no haber aprendido sino, en todo caso, por algo distinto. La puntuación de este ítem fue de solo el 2,41, de las más bajas. Aprender es un objetivo de la enseñanza y su no consecución podría comportar frustración. Si no se ha sentido, puede ser o bien porque se ha aprendido (teniendo en cuenta que en el aprendizaje colaborativo se aprende más de lo que aprendería cada estudiante por separado, por todos Guitert y Giménez, 2000), o porque aprender no era un objetivo de los estudiantes stricto sensu. Para dilucidar esta disyuntiva hubiese sido útil preguntar directamente a los estudiantes cuales son sus objetivos al hacer la asignatura, para evitar toda duda al respecto. Sin embargo cabe asumir que esos objetivos en el caso del trabajo colaborativo son dos: aprender, y contribuir a que los compañeros también lo hagan (Pujolàs, 2008b). Los alumnos que están rechazando el aprendizaje colaborativo pese a considerar que han aprendido, posiblemente ignoren que habrían aprendido aún menos si hubiesen trabajado individualmente (Olsen y Kagan, 1992). Además, es posible que la frustración haya, incluso, contribuido a que hayan aprendido. Como señalan algunos autores, hay alumnos que después de haber experimentado frustración han incrementado su ritmo de trabajo (Bachour, Kaplan y Dillenbourg, 2008). Por ende, la frustración no necesariamente es mala, sino que puede operar como catalizador de un mayor aprendizaje. Incluso, puede aprenderse algo de la propia frustración. Por ejemplo, puede dar pie a la comunicación con los otros miembros del grupo para buscar estrategias sobre cómo gestionar esa sensación, en especial formas alternativas de enfocar el trabajo (en esta línea, en los estudios de geometría, Bjuland, 2004). Al mismo tiempo, este hallazgo sugiere que es importante que el profesor que hace el seguimiento de la actividad esté pendiente ante posibles brotes de frustración, para animar el debate en ese sentido. En el mejor de los casos, el profesor puede prever en qué momentos se van a sentir frustrados los alumnos, para emprender la acción que corresponda (como sugiere, en relación con ejercicios de matemáticas por ordenador, Kim, 2012). De hecho, si se está comenzando a programar a ordenadores para que lo hagan (Klein, Moon y Picard, 2002), también lo deberían poder hacer los profesores.

\section{6.- CONCLUSIONES}

El empleo de una herramienta wiki en una asignatura de Derecho en una universidad eminentemente presencial arrojó resultados contrapuestos. Mientras que una parte de los alumnos la recibieron de modo positivo, otros la percibieron como una dificultad añadida o incluso como una pérdida de tiempo. Parte de los estudiantes encontraron que las wikis suponen una herramienta innecesaria o respecto de la cual hay alternativas mejores.

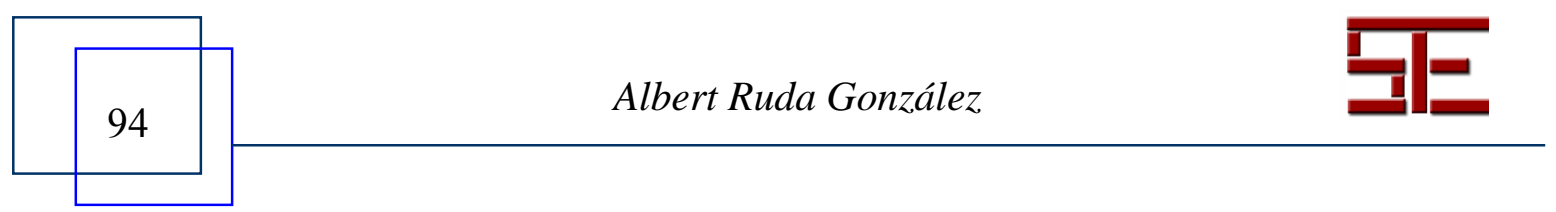




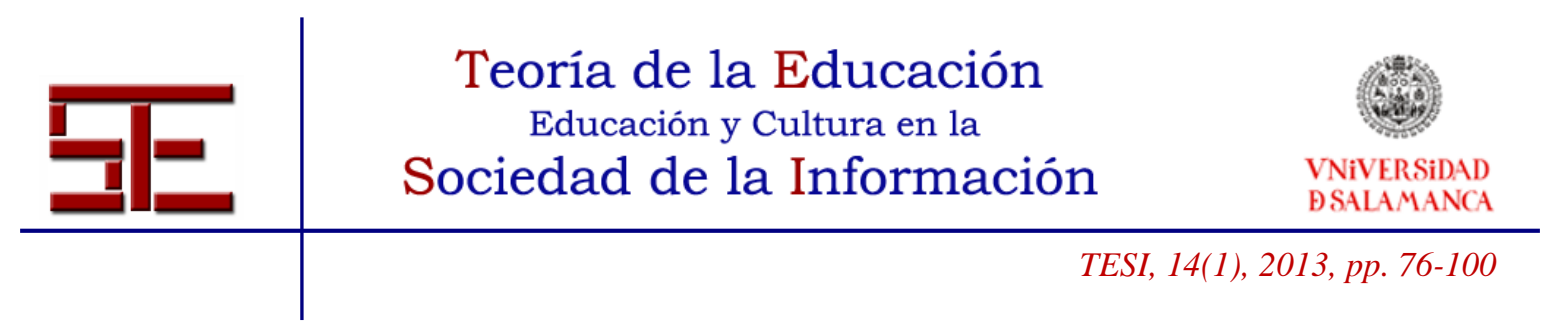

Marcadamente, muchos destacaron que no necesitaban esa herramienta para realizar un trabajo de tipo colaborativo, ya que podían reunirse y trabajar en persona, incluso, sentándose todos los miembros de un grupo ante el ordenador para confeccionar el trabajo con un solo usuario informático, común a todos ellos. Por tanto, la falta de tiempo y el empleo de la wiki son dos factores que generan frustración de los estudiantes en un porcentaje relevante. La wiki se lleva la palma al ser el factor más frustrante, según el cuestionario. Los comentarios realizados por los estudiantes confirman ese resultado, así como la sesión de grupo focal y los problemas con el aprendizaje de su funcionamiento, que motivan la mayoría de comunicaciones con el profesor durante el desarrollo de la actividad.

Además, es otra fuente de frustración la falta de colaboración de algunos miembros de los grupos. Sin embargo, ese motivo no aparece en las comunicaciones que se hacen en los espacios de debate, foros, etc., sino que cuando aflora es en la comunicación directa con el profesor (en persona, en el caso del grupo más conflictivo y único donde hubo una escisión provocada por dicho problema), y en el cuestionario. Los alumnos ahondan en este motivo en sus comentarios en los espacios ad hoc del cuestionario, y expresan repetidamente que sería injusto que todos los alumnos obtuviesen la misma nota habida cuenta de la contribución desigual al trabajo. Notablemente, la falta de colaboración no es el motivo principal de frustración (ya se ha visto que lo es el empleo de la wiki), aunque sí uno de los más importantes.

El trabajo colaborativo mediante wikis en unos estudios eminentemente presenciales requiere una gran dosis de planificación por el profesorado, preparación previa de los estudiantes para que conozcan la herramienta y prestar gran atención a la respuesta de éstos para evitar o anticipar su frustración, así como flexibilidad para corregir posibles imprevistos o problemas de los estudiantes. El tiempo dedicado a las wikis puede ser percibido por éstos como pérdida de tiempo, por lo cual debe explicarse muy bien el sentido de esa elección. Además hay que controlar que las aportaciones de los estudiantes sean lo más equilibradas posible dentro de los grupos. No solo el esfuerzo desigual, sino la falta de tiempo, son factores de frustración claves en la enseñanza mixta o blended learning. Un planteamiento claro y realista de los objetivos del CSCL posiblemente pueda ayudar a disminuir esa frustración.

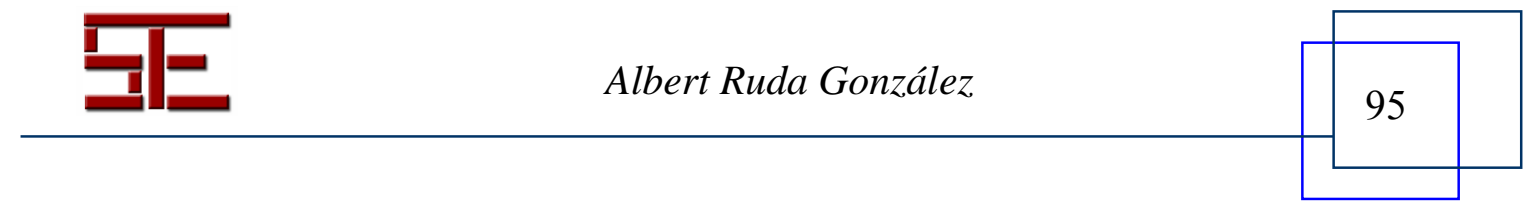




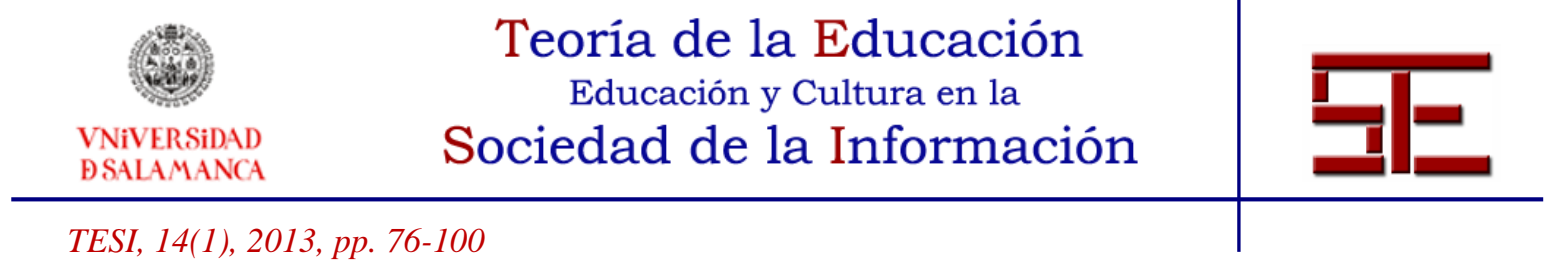

En fin, puede sugerirse que es preciso estudiar mejor la relación entre los motivos de la frustración percibida por los estudiantes en contextos distintos en los que se lleva a cabo el aprendizaje colaborativo. En particular habría que estudiar la relación entre la falta de tiempo y la dificultad de la tarea, y dilucidar si realmente el desequilibrio entre las dedicaciones respectivas es tan decisivo como sugiere la literatura anterior (Capdeferro y Romero, 2012) o es más bien, como aquí se ha sugerido, uno más entre otros motivos con casi el mismo peso. Mejorar la comprensión sobre la frustración puede ayudar a proporcionar a los estudiantes una experiencia mejor con el aprendizaje colaborativo y a anticipar posibles dificultades en la ejecución de las actividades.

\section{7.- BIBLIOGRAFÍA}

Anderson, T. y Kanuka, H. (2007). Investigación en e-learning. Antología de textos del libro e-Research Methods, Strategies, and Issues (2003). Barcelona: UOC.

Allen, I. E. \& Seaman, J. (2007). Online nation: Five years of growth in online learning. Needham, MA: Sloan Consortium.

Andrews, S. (1998). Problems Students Encounter during Math Instruction in MixedAbility Classrooms. ERIC (Educational Resource Information Center). Master's Action Research Project, Saint Xavier University and IRI/Skylight.

Artino, A. R. (2008, March). Understanding satisfaction and continuing motivation in an online course: An extension of social cognitive, control-value theory. Paper presented at the annual meeting of the American Educational Research Association, New York, NY.

Bachour, K.. Kaplan, F. y Dillenbourg, P. (2008). Reflect: An Interactive Table for Regulating Face-to-Face Collaborative Learning. En Dillenbourg, P. y Specht, M. (eds.): EC-TEL 2008, (pp. 39-48). LNCS 5192.

Baltes, B. B., Dickson, M. W., Sherman, M. P., Bauer, C. C. \& LaGanke, J. S. (2002). Computer mediated communication and group decision making: A meta-analysis. Organizational Behavior and Human Decision Processes, 87, 156-179.

Berkowitz, L. (1989). Frustration-Aggression Hypothesis: Examination and Reformulation. Psychological Bulletin, 106 (1), 59-73.

Bessiere, K., Newhagen, J. E., Robinson, J. P. \& Shneiderman, B. (2004). A model for computer frustration: The role of instrumental and dispositional factors on incident,

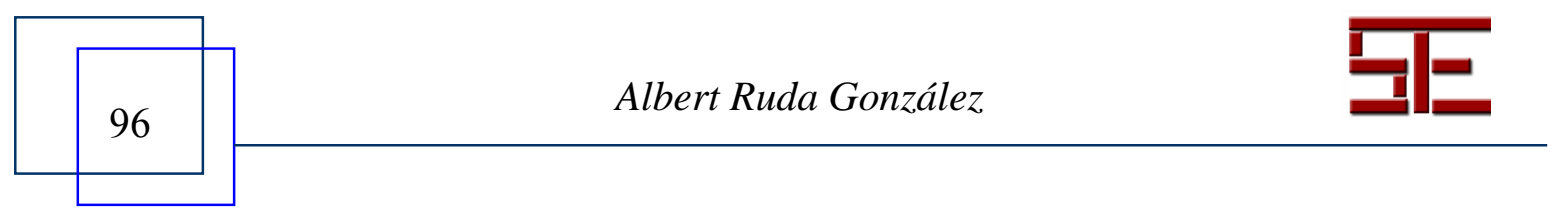




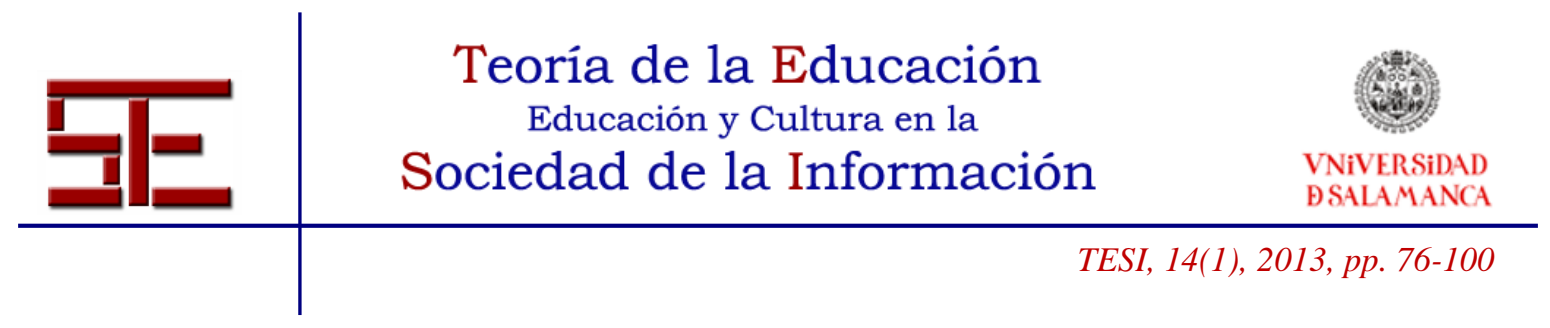

session, and post-session frustration and mood. Computers in Human Behavior, 22 (6), 941-961

Bjuland, R. (2004). Student teachers' reflections on their learning process through collaborative problem solving in Geometry. Educational Studies in Mathematics, 55, 199225 .

Borges, F. (2005). La frustració de l'estudiant en línia. Causes i accions preventives. Revista electrònica dels Estudis d'Humanitats i Filologia de la UOC, 7, 1-9.

Brindley, J. E., Walti, C. \& Blaschke, L. M. (2009). Creating effective collaborative learning groups in an online environment. International Review of Research in Open and Distance Learning, 10 (3), 1-18.

Bryman, A. (2008). E-research: Using the Internet as object and method of data collection. En A. Bryman, Social research methods (3rd ed., pp. 627-659). Oxford: Oxford University Press.

Burdett, J., \& Hastie, B. (2009). Predicting satisfaction with group work assignments. Journal of University Teaching \& Learning Practice, 6 (1), 61-71.

Capdeferro, N., y Romero, M. (2012). Are Online Learners Frustrated with Collaborative Learning Experiences? International Review of Research in Open and Distance Learning, $13(2), 26-43$.

Chapman, K. J., \& van Auken, S. (2001). Creating positive group project experiences: An examination of the role of the instructor on students' perceptions of group projects. Journal of Marketing Education, 22 (2), 117-127.

Chóliz Montañés, M. (2004). Psicología de la Motivación: el proceso motivacional. Valencia: Universidad de Valencia. Obtenido el 27.4.2012 desde: http://www.uv.es/ choliz.

Conrad, D. L. (2002). Engagement, excitement, anxiety, and fear: Learners' experiences of starting an online course. American Journal of Distance Education, 16 (4), 205-226.

Creswell, J. W. (2004). Educational research. Planning, conducting and evaluating quantitative and qualitative research ( $2^{\mathrm{a}}$ ed.). Boston: Pearson Prentice-Hall

Duart, J. M., y Sangrà, A. (2000). Aprender en la virtualidad. Barcelona: Edicions de la Universitat Oberta de Catalunya, Gedisa.

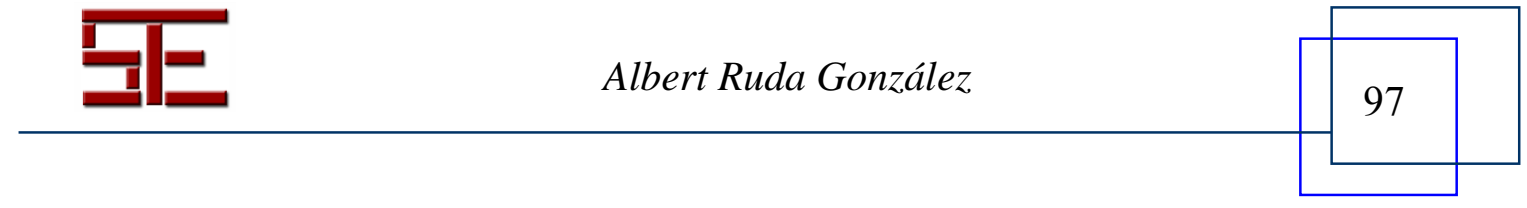




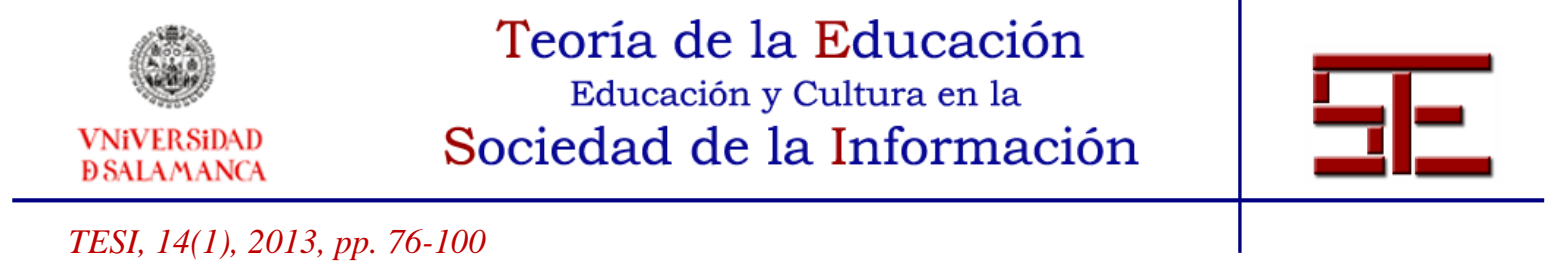

Echeita, G. (2003). Sentir el apoyo de los compañeros. Las estrategias de aprendizaje coope-rativo. En Barnett, L. et al. Motivación, tratamiento de la diversidad y rendimiento académico: el aprendizaje cooperativo. (pp. 33-37). Barcelona: Graó.

Flick, U. (2006). Qualitative online research: Using the internet. En U. Flick, An introduction to qualitative research (3rd ed., pp. 255-270). London: Sage.

Fortner, R. W. (2002). Chapter 5: Cooperative learning: a basic instructional methodology for global science literacy (pp. 79-92). En Mayer, V. J. (Ed.), Global Science Literacy. Deventer: Kluwer Academic Publishers.

Goikoetxea, E. y Pascual, G. (2002). Aprendizaje cooperativo: bases teóricas y hallazgos empíricos que explican su eficacia. Universidad de Deusto, Educación XX, (pp. 227-247).

Goold, A., Craig, A. \& Coldwell, J. (2008). The student experience of working in teams online. Proceedings ascilite Melbourne 2008.

Grinter, R. E., Herbsleb, J. D. \& Perry, D. E. (1999, November). The geography of coordination: Dealing with distance in R\&D work. Proceedings, ACM Conference on Supporting Group Work (GROUP 99), Phoenix, AZ (pp. 306-315).

Guitert, M. y Giménez, F. (2000). Aprendizaje cooperativo en entornos virtuales. En Duart, J.M., y Sangrà, A. Aprender en la virtualidad. Barcelona: Edicions de la Universitat Oberta de Catalunya, Gedisa.

Järvenoja, H., \& Järvelä, S. (2005). How students describe the sources of their emotional and motivational experiences during the learning process: A qualitative approach. Learning, 15, 465-480.

Johnson, D. W.; Johnson, R. E. y Holubec, E. H. (1999). El aprendizaje cooperativo en el aula. Buenos Aires (etc.): Paidós.

Kamenetzky, G. V., Cuenya, L., Elgier, A. M., López Seal, F., Fosacheca, S., Martin, L. y Mustaca, A. E. (2009). Respuestas de Frustración en Humanos. Terapia Psicológica, 27 (2), 191-201.

Kim, Ch. (2012). The role of affective and motivational factors in designing personalized learning environments. Educational Technology Research and Development, 60 (4), 563 584.

Klein, J.; Moon, Y., y Picard, R. W. (2002). This computer responds to user frustration: Theory, design, and results. Interacting with Computer, 14, 119-140.

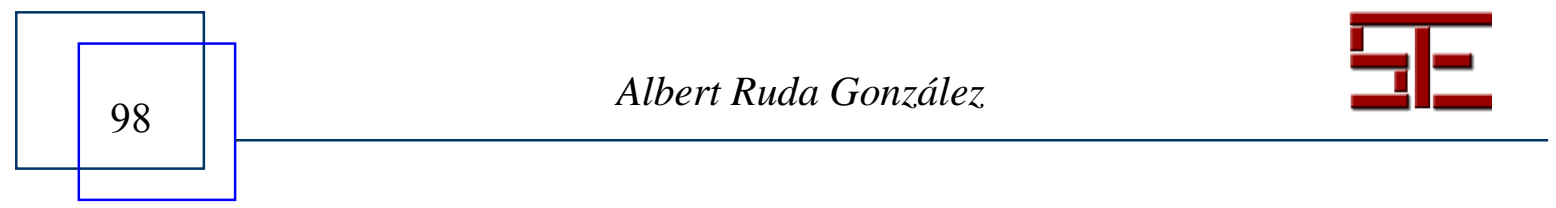




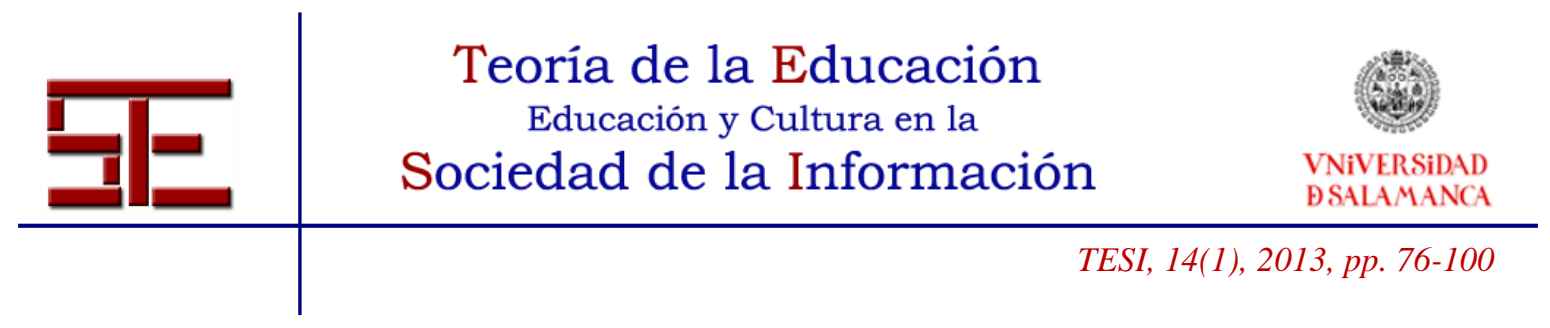

Kreijns, K., Kirschner, P. A., Jochems, W., \& Buuren, H. (2004). Determining sociability, social space, and social presence in (a)synchronous collaborative groups. Cyberpsychology \& Behavior, 7 (2), 155-172.

Liu, M.; Horton, L.; Olmanson, J. y Toprac, P. (2011). A study of learning and motivation in a new media enriched environment for middle school science. Educational Technology Research and Development, 59, 249-265.

Melton, B., Graf, H. y Chopak-Foss, J. (2009). Achievement and Satisfaction in Blended Learning versus Traditional General Health Course Designs. International Journal for the Scholarship of Teaching and Learning, 3 (1), 1-13.

Monereo Font, C. y Duran Gisbert, D. (2001). Entramats: mètodes d'aprenentatge cooperatiu i col-laboratiu. Barcelona: Edebé

Naidu, S. y Oliver, M. (1999). Critical incident-based computer supported collaborative learning. Instructional Science, 27, 329-354.

Neuman, W. L. (2006). Qualitative and quantitative research designs. En W. L. Neuman, Social research methods: Qualitative and quantitative approaches (6th ed., pp. 149-178). Boston: Pearson Prentice-Hall.

Olsen, R. E. W-B. y Kagan, S. (1992). About Cooperative Learning. En C. Kessler. Cooperative language learning: a teacher's resource book. Englewood Cliffs: Prentice Hall Regents.

Ovejero Bernal, A. (1990). El Aprendizaje cooperativo: una alternativa eficaz a la enseñanza tradicional. Barcelona: PPU.

Pujolàs Maset, P. (2008). El Aprendizaje cooperativo: 9 ideas clave. Barcelona: Graó.

Rebollo Catalán, M. A., García Pérez, R., Barragán Sánchez, R., Buzón García, O. y Vega Caro, L. (2008). Las emociones en el aprendizaje online. RELIEVE, 14 (1). Obtenido el 17.4.2012 desde http://www.uv.es/RELIEVE/v14n1/RELIEVEv14n1_2.htm

Rodríguez, D. \& Valldeoriola, J. (2009). Metodologia de la investigació. Barcelona: UOC.

Sela, O. (2010). The Power of the Model: One Step towards Developing Blended Learning Courses in Higher Education. MERLOT Journal of Online Learning and Teaching, 6 (4), 820-827.

Sharan, Y. y Sharan, S. (2004). El Desarrollo del aprendizaje cooperativo a través de la investigación en grupo. Sevilla: M.C.E.P.

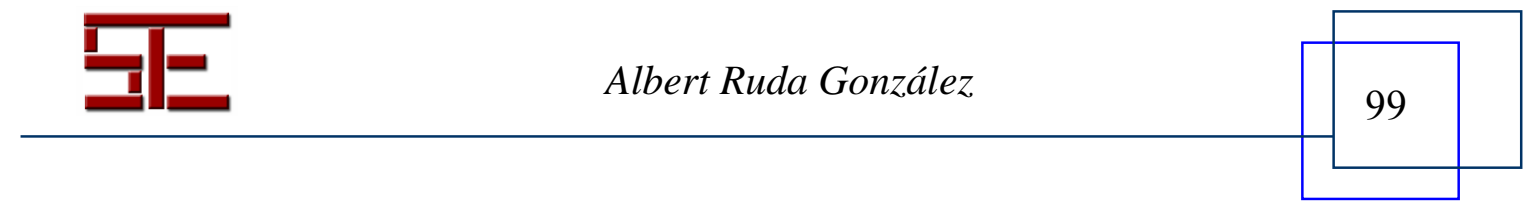




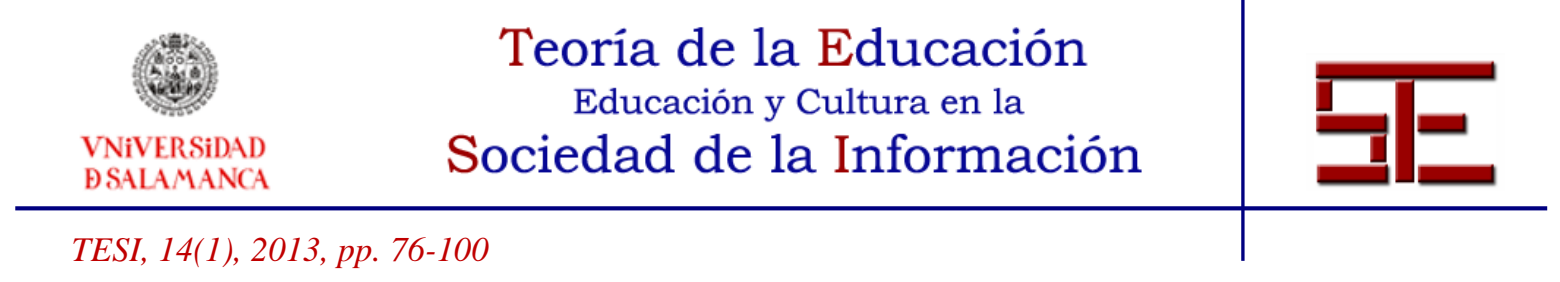

Suárez Guerrero, C. (2010). Aprendizaje cooperativo e interacción asíncrona textual en contextos educativos virtuales. Píxel-Bit. Revista de Medios y Educación, 36, 53-67.

Úriz Bidegáin, N. (Coord.) (1999). Aprendizaje cooperativo, Educación Primaria. Pamplona: Departamento de Educación y Cultura, Gobierno de Navarra, Dpto. Presidencia e Interior. Obtenido el 1.11.2011 desde: http://dpto.educacion.navarra.es/publicaciones/pdf/apr_coop.pdf.

Para citar el presente artículo puede utilizar la siguiente referencia:

Ruda González, A. (2013). Percepción de los estudiantes sobre el uso de wiki. Análisis de una experiencia de aprendizaje colaborativo en la Universitat de Girona. Revista Teoría de la Educación: Educación y Cultura en la Sociedad de la Información. 14(1), 76-100 [Fecha de consulta: dd/mm/aaaa].

http://campus.usal.es/ revistas_trabajo/index.php/revistatesi/article/view/9444/9733 